\title{
Theory of positron production in heavy-ion collisions
}

\author{
Joachim Reinhardt \\ Institut für Theoretische Physik, Johann Wolfgang Goethe Universität, 6000 Frankfurt am Main, West Germany \\ Berndt Müller* and Walter Greiner* \\ Physics Division, Oak Ridge National Laboratory, Oak Ridge, Tennessee 37830 \\ and Department of Physics and Astronomy, Nuclear Physics Division, Vanderbilt University, Nashville, Tennessee 37203
}

(Received 1 December 1980)

\begin{abstract}
Collisions of very heavy ions at energies close to the Coulomb barrier are discussed as a unique tool to study the behavior of the electron-positron field in the presence of strong external electromagnetic fields. To calculate the excitation processes induced by the collision dynamics, a semiclassical model is employed and adapted to describe the field-theoretical many-particle system. An expansion in the adiabatic molecular basis is chosen. Energies and matrix elements are calculated using the monopole approximation. In a supercritical $\left(Z_{1}+Z_{2} \gtrsim 173\right)$ quasiatomic system the $1 s$ level joins the antiparticle continuum and becomes a resonance, rendering the neutral vacuum state unstable. Several methods of treating the corresponding time-dependent problem are discussed. A projectionoperator technique is introduced for a fully dynamical treatment of the resonance. Positron excitation rates in $s_{1 / 2}$ and $p_{1 / 2}$ states are obtained by numerical solution of the coupled-channel equations and are compared with results from first- plus second-order perturbation theory. Calculations are performed for subcritical and supercritical collisions of $\mathrm{Pb}-\mathrm{Pb}, \mathrm{Pb}-\mathrm{U}, \mathrm{U}-\mathrm{U}$, and $\mathrm{U}-\mathrm{Cf}$. Strong relativistic deformations of the wave functions and the growing contributions from inner-shell bound states lead to a very steep $Z$ dependence of positron production. The results are compared with available data from experiments done at GSI. Correlations between electrons and positrons are briefly discussed.
\end{abstract}

\section{INTRODUCTION}

The peculiarities of the behavior of electrons in strong external electromagnetic fields ${ }^{1-7}$ have attracted continuous interest ever since the beginnings of relativistic quantum mechanics. The anomalous behavior of reflection and transmission coefficients for electrons incident on a potential barrier higher than $2 m c^{2}$ became known as Klein's paradox. ${ }^{8}$ Responsible for this effect is the mixing between positive- and negative-frequency solutions which leads to the creation of electron-positron pairs..$^{9-12}$ This is most simply understood in $\mathrm{Di}-$ rac's hole picture: An electron from the totally occupied negative continuum can be set free by tunneling through the gap, leaving a hole, i.e., positron behind. Strong electric fields which extend over a sufficiently large area of space can continuously produce pairs. ${ }^{13}$

Related to this phenomenon is the problem of a strong and localized potential well, the physics of which has been fully understood for only a decade. Let us think of the stationary potential well produced by the Coulomb field of an extended heavy nucleus. With increasing strength of the potential the energies of all bound states decrease steadily. At $Z \simeq 150$ (assuming normal nuclear density) the $1 s$ state obtains negative total energy and at $Z_{\mathrm{cr}}$ $\simeq 172$ (Refs. 10 and 14-16) it enters the negative energy continuum $E_{1 s}<-m c^{2}$. At this point the spectrum of eigenstates of the Dirac equation is subject to a characteristic change. The $1 s$ state becomes a resonance, which decays spontaneously by emission of two (due to spin degeneracy) positrons if it were prepared empty. The new stable ground state of the system consists of the nucleus plus two electrons in the $K$ shell; it is called the charged vacuum. ${ }^{17,18}$ The experimental exploration of this new phenomenon would constitute an important test of the theory of quantum electrodynamics (QED) in the region of strong fields.

Interest in this area was nourished by speculations on the existence of superheavy nuclei. Unfortunately nuclei with sufficiently high charge ( $Z>172$ for normal density, $Z>137$ for pointlike charge) have not been found and probably do not exist. The only known way to assemble a supercritical charge at least for a limited period of time is in collisions of very heavy ions, where charges up to $Z_{1}+Z_{2} \simeq 190$ can be reached. In such scattering experiments, however, the dynamics of the collision becomes extremely important. The time scale must be sufficiently long to allow the electrons (positrons) to adjust to the variation of the combined Coulomb field of the two nuclei. Since typical velocities required to bring the nuclei closely together are about $v / c \simeq 0.1$, an adiabatic description is meaningful only for fast moving electrons. When the total nuclear charge exceeds the inverse fine-structure constant $\left(Z_{1}+Z_{2}\right) \alpha>1$ the electronic wave functions are very sensitive to the internuclear distance $R(t)$. This is related to the well-known singularity of the solutions of the relativistic point-nucleus 
problem at $Z=1 / \alpha \simeq 137$, where the wave functions of all $s_{1 / 2}$ and $p_{1 / 2}$ states are found to collapse (for a further discussion of this problem see also Ref. 19). Owing to this effect the nuclear motion induces strong excitations which give rise to the formation of inner-shell holes and to the emission of $\delta$ electrons and positrons.

Over the last several years various aspects of the excitation process in collisions of very highly charged systems have been investigated. The results of recent experiments, mostly performed at GSI (Darmstadt), are in general agreement with theoretical predictions. In the present article we will concentrate on positron creation. In particular, our aim is to give an adequate description of this process in collisions of very heavy ions. Pair creation in charged-particle collisions has been extensively investigated using Born approximation or the Weizsäcker-Williams approximation which are applicable to collisions of light particles or at highly relativistic energies. In the case of heavy particles colliding at "low" velocities, however, the use of the Born approximation is unjustified. This was demonstrated by the experimental disproof of the validity of the HeitlerNordheim formula ${ }^{20,21}$ in proton-nucleus collisions. ${ }^{22,23}$ As shown in Refs. 24 and 25 the nuclear Coulomb repulsion greatly reduces pair cross sections.

Even more important for our problem is the correct treatment of the distortion of electron and positron states in the Coulomb field of the two nuclei as discussed above. As a consequence, the rate for direct pair production grows very rapidly with increasing nuclear charge in the superheavy region. This has to be compared with the $Z_{1}^{2} Z_{2}^{2}$ dependence deduced from the lowest-order Feynman diagram. Furthermore the role of innershell bound states (in particular $1 s$ and $2 p_{1 / 2}$ ) becomes increasingly important if $Z_{1}+Z_{2}$ approaches $Z_{\mathrm{cr}}$. These may act as intermediate states in multistep excitations or (in the still hypothetical collisions of naked nuclei) be the dominant final states for the created electron.

In the following we will first describe the quasimolecular model for electronic excitations using the independent-electron approximation. Properties of the resulting amplitudes are discussed and their use in the calculation of pair creation is demonstrated. In Sec. III we will discuss the special problems arising in the case of supercritical collisions $\left(Z_{1}+Z_{2}>Z_{\mathrm{cr}}\right)$. We introduce a method to treat the time-dependent resonance which is based on a projection-operator technique (Sec. IV). Preliminary accounts of this theory have been given in Refs. 26 and 27. The final sections contain details of the model employed and the numerical results for positron creation in several collision systems, which are compared with currently available experimental data. If not stated otherwise, we will use natural units, $\hbar=m_{e}$ $=c=1$, i.e., energies are measured in multiples of $511.004 \mathrm{keV}$ and lengths in multiples of the Compton wavelength of the electron $386.159 \mathrm{fm}$.

\section{ELECTRONIC EXCITATIONS IN THE ADIABATIC PICTURE}

A vast number of methods and approximation schemes has been developed to calculate electronic-excitation processes in atomic collisions (see, e.g., Refs. 28-30). Until recently the theory of excitations in collisions of very heavy ions has received comparatively little attention due to computational difficulties and lack of experimental data. In the present work we are interested in the creation of positrons in collisions of very heavy ions at energies comparable to the nuclear Coulomb barrier. As implied already in the motivation given in the first section, these collisions are characterized by the coherent action of the combined nuclear Coulomb centers. Under these conditions first-order perturbational calculations are not sufficient. In particular, a correct description of inner-shell bound states becomes essential. Therefore we have to develop a theory which treats electronic bound states and positrons in a unified manner and allows for multiple excitations.

The nuclear motion will be treated classically throughout, since the Bohr-Sommerfeld parameter $\eta=Z_{1} Z_{2} e^{2} / \hbar v$ is very large compared to unity for the envisaged systems. Furthermore the energy transferred to the electron-positron field can be neglected compared to the nuclear kinetic energy. In the semiclassical approximation the nuclei are treated as sources of a time-dependent external potential. Since the interesting excitations occur predominantly at small internuclear distances, Rutherford trajectories have to be used to describe the nuclear motion. As usual, the electronic wave function is expanded in a complete set of basis states. The scattering problem thereby is reduced to an infinite system of coupled differential equations in time, which may be solved numerically after truncation of the basis. For electrons moving relativistically the nuclear motion is "slow", $v / c \lessgtr 0.1$, so that an adiabatic basis set of molecular two-center Dirac (TCD) solutions will lead to the best convergence. The actual calculations will be performed using the monopole approximation to the two-center wave functions.

In the following we will write the coupled differ- 
ential equations governing the time development of the one-electron occupation amplitudes. Next, various useful symmetry relations between these amplitudes are discussed. Finally, using the language of second quantization, we will demonstrate how the single-particle amplitudes are related to production rates of electrons, holes, and positrons.

\section{A. The coupled-channel equations}

The wave function of a single electron moving in the externally prescribed time-dependent electromagnetic field generated by the colliding nuclei is determined by the Dirac equation

$$
i \hbar \frac{\partial}{\partial t} \Phi_{i}(t)=H_{\mathrm{TCD}}(\overrightarrow{\mathrm{R}}(t)) \Phi_{i}(t),
$$

with the two-center Hamiltonian

$$
H_{\mathrm{TCD}}=\vec{\alpha} \cdot[\overrightarrow{\mathrm{p}}-e \overrightarrow{\mathrm{A}}(\overrightarrow{\mathrm{r}}, \overrightarrow{\mathrm{R}}(t))]+\mathrm{eV}(\overrightarrow{\mathrm{r}}, \overrightarrow{\mathrm{R}}(t))+\beta m .
$$

If the electron occupies a definite atomic state $\phi_{i}$ before the collision, Eq. (2.1) has to be solved with the boundary condition $\Phi_{i}^{(+)}(t \rightarrow-\infty) \rightarrow \phi_{i}$. The final amplitude for the excitation of a particular state is given by the overlap of its wave function with $\Phi_{i}^{(+)}(t \rightarrow \infty)$. In the absence of incident photons the electromagnetic potential $(\vec{A}, V)$ can be calculated from the current generated by the moving nuclei, i.e.,

$$
A_{\mu}(\overrightarrow{\mathrm{r}}, t)=\int D^{R}\left(\overrightarrow{\mathrm{r}}, t ; \overrightarrow{\mathrm{r}}^{\prime}, t^{\prime}\right) j_{\mu}\left(\overrightarrow{\mathrm{r}}^{\prime}, t^{\prime}\right) d^{3} r^{\prime} d t^{\prime},
$$

where $D^{R}$ is the retarded Green's function of the wave equation. In the Coulomb gauge the timelike component reduces to the instantaneous interaction

$$
V(\overrightarrow{\mathrm{r}}, t)=-\frac{Z_{1} e}{\left|\overrightarrow{\mathrm{r}}-\overrightarrow{\mathrm{R}}_{1}(t)\right|}-\frac{Z_{2} e}{\left|\overrightarrow{\mathrm{r}}-\overrightarrow{\mathrm{R}}_{2}(t)\right|}
$$

assuming, for shortness, point nuclei. The magnetic $^{31}$ and retardation ${ }^{32}$ effects contained in the vector potential $\vec{A}$ will be neglected since they are of higher order in $v / c$. A further investigation of $\vec{A}$ in the Coulomb gauge and the resulting polarization effects has been given in Ref. 33 .

A direct integration of the dynamical two-center Dirac problem analogous to the case of $p-H$ collisions for the Schrödinger equation ${ }^{34}$ up to now has not been attempted. It would be very demanding numerically, particularly if one were interested in the energy spectra of emitted particles. Instead, the time-dependent wave function $\Phi_{i}(t)$ is expanded in some complete set of basis states $\phi_{k}(t)$

$$
\Phi_{i}(t)=\sum_{k} a_{i k}(t) \phi_{k}(t) e^{-i X_{k}(t)} .
$$

The summation here and in the following is understood to include integration over the continuous parts of the spectrum. The phase factor $\chi_{k}(t)$ is conveniently chosen so as to eliminate the diagonal matrix element of the Hamiltonian, i.e.,

$$
\chi_{k}(t)=\int_{t_{0}}^{t} d t^{\prime}\left\langle\phi_{k}\left(t^{\prime}\right)|H(\vec{R}(t))| \phi_{k}\left(t^{\prime}\right)\right\rangle .
$$

The value of $t_{0}$ is arbitrary, it defines the overall phase of the amplitudes.

The resulting system of coupled differential equations for the expansion amplitudes equivalent to $(2.1)$ is

$\dot{a}_{i j}=-\sum_{k \neq j} a_{i k}\left\langle\phi_{j}|\partial / \partial t+i H| \phi_{k}(t)\right\rangle e^{-i\left(x_{k}-x_{j}\right)}$.

In general, therefore, excitations are caused by two kinds of coupling operators: $\partial / \partial t$ acting on the parametric time dependence of the wave function, and $H$ which may be nondiagonal in the basis $\phi_{k}$. The time-derivative operator in (2.7) may be split in a radial and a rotational part $\partial / \partial t \rightarrow \dot{R} \partial / \partial R$ $-i \vec{\omega} \cdot \vec{j}$, where $\vec{j}$ is the electronic angular momentum operator and $\vec{\omega}$ the angular velocity of the internuclear axis.

If the basis set is nonorthogonal, (2.7) is modified to

$$
\begin{gathered}
\sum_{k}\left\langle\phi_{j} \mid \phi_{k}\right\rangle e^{-i\left(x_{k}-x_{j}\right)} \dot{a}_{i k} \\
=-\sum_{k} a_{i k}\left(e^{\left.-i \alpha_{k}-x_{j}\right)}\left\langle\phi_{j}|\partial / \partial t+i H| \phi_{k}\right\rangle\right. \\
\left.-i\left\langle\phi_{j} \mid \phi_{k}\right\rangle\left\langle\phi_{k}|H| \phi_{k}\right\rangle\right)
\end{gathered}
$$

To solve for $\dot{a}_{i k}$ the coupling matrix has to be multiplied by the inverse of the overlap matrix.

While in principle the set of equations (2.7) is still exact, it can be solved only by approximation methods so that the outcome of practical calculations depends critically on the chosen basis $\phi_{k}$. Two special choices have been widely used.

(1) The atomic picture where the eigenstates of the target atom are disturbed by the time-dependent Coulomb field of the passing projectile. Except for recoil effects only potential coupling is present. The SCA (semiclassical approximation) model, which was pioneered by Bang and Hansteen, ${ }^{24}$ has been successful in describing highly asymmetric collisions. For slow or symmetric collisions the adiabatic relaxation of the wave functions becomes important and can be included only approximately by binding-energy and polarization corrections. A model for symmetric collisions was proposed by Briggs, ${ }^{35}$ who uses the stationary states of the united atom limit as a basis. 
(2) In slow near-symmetric collisions the quasimolecular picture becomes applicable. The basis consists of the eigenstates of the stationary twocenter problem

$$
H_{\mathrm{TCD}}(\overrightarrow{\mathrm{R}}) \phi_{k}=E_{k}(R) \phi_{k}
$$

evaluated at each internuclear distance. The couplings in the PSS (perturbed stationary state) model are solely due to the $\partial / \partial t$ operator; as discussed at length in Sec. IV, this will change in supercritical collision. There the adiabatic $1 s$ state becomes unstable even without the imposition of an external time dependence.

In the present work we are interested in close collisions of very heavy systems. The nuclear motion can be regarded as slow compared to the relativistic velocities of the electrons in the innermost bound states, ${ }^{36}$ which speaks in favor of the adiabatic picture. A more detailed study of this problem is given in Ref. 37 where the "optimal" basis having a minimal excitation strength is found to follow closely the adiabatic basis.

\section{B. Properties of the excitation amplitudes}

The amplitudes $a_{i j}(t)$ describing the transition of an electron from state $i$ to state $j$ in the course of the collision satisfy several useful symmetry relations. These may be used for the reduction of computational effort and for checks of the numerical accuracy. All relations given are exact if the solutions of the coupled differential equations are inserted, irrespective of the choice and size of the basis. We start from the observation that the coupling matrix

$$
M_{j k} \equiv\left\langle\phi_{j}|\partial / \partial t+i H| \phi_{k}\right\rangle
$$

entering (2.7) is anti-Hermitian (if an orthonormal basis set is used):

$$
M_{j k}=-M_{k j}^{*} \text {. }
$$

(i) The orthonormality condition

$$
\sum_{k} a_{j k}^{*}(t) a_{i k}(t)=\delta_{i j}
$$

can be verified by differentiation with respect to time and use of (2.7) and (2.10). This condition implies the orthogonality of the set of wave functions $\Phi_{i}$ at every instant of time

$$
\left\langle\Phi_{i}(t) \mid \Phi_{j}(t)\right\rangle=\delta_{i j},
$$

which could have been expected from the unitarity of the time-development operator.

(ii) The identity

$$
\sum_{k} a_{k j}^{*}(t) a_{k i}(t)=\delta_{i j}
$$

can be deduced in a similar way. It guarantees the completeness of the set $\Phi_{k}$ at any time if one starts from a complete basis $\phi_{k}$.

(iii) In a time-symmetric collision one has also

$$
a_{i j}(\infty)=a_{j i}(\infty) \text {. }
$$

In contrast to (2.12) and (2.13) this identity is valid only in the limit $t \rightarrow \infty$. It reflects the principle of detailed balance which equates the transition rates in both directions of a given reaction if the interaction is invariant under time reflection. Thus (2.14) is valid only for collisions with a symmetric nuclear trajectory, i.e., $R(t)=R(-t)$ and $\vec{\omega}(t)=\vec{\omega}(-t)$ which holds for Coulomb scattering neglecting loss of energy and angular momentum. In this case the coupling matrix elements between the monopole basis states discussed below satisfy

$$
M_{i j}(t)=-M_{i j}^{*}(-t),
$$

which leads to (2.14) if inserted in the complex conjugate of the differential equation (2.7). In general (2.15) is correct only up to a phase factor and (2.14) holds only for the absolute values. ${ }^{38}$ This restriction applies also if the phases $\chi_{i}(t)$ are not chosen symmetric with respect to $t=0$.

$$
\text { (iv) } a_{i j}(\infty)=\sum_{k} a_{i k}(0) a_{j k}(0) \text {. }
$$

This equation holds under the same conditions as (2.14). It can be derived using the time-development operator defined by $a_{i j}(t)=W_{j k}\left(t, t_{0}\right) a_{i k}\left(t_{0}\right)$. Because of (2.15) and (2.7), $W$ satisfies the symmetry relation

$$
W_{j k}(t, 0)=W_{j k}^{*}(-t, 0),
$$

which together with the unitarity condition

$$
W^{-1}\left(t, t_{0}\right)=W\left(t_{0}, t\right)=W^{\dagger}\left(t, t_{0}\right)
$$

and the identification $W_{i j}(0,-\infty)=a_{j i}(0)$, proves (2.16). The identity shows that it is sufficient to calculate the excitation amplitudes $a_{i j}(0)$ for the incoming branch of the trajectory only. Equation (2.16) is immediately generalized for timeasymmetric collisions; the sets $a_{i k}(0)$ and $a_{j k}(0)$ then have to be calculated for different kinematics. An extension of this formalism and its use for the calculation of electronic excitations in deep inelastic collisions and for muon-induced fission recently has been discussed by Müller and Oberacker. ${ }^{39}$

\section{Excitations of the many-electron system}

The discussion up to now was concerned with single-electron excitations only. This is clearly 
insufficient since many atomic electrons are usually present at the beginning of a heavy-ion collision. Furthermore, if pair creation is to be described the antiparticle continuum must be treated properly. In Dirac's hole picture all states of the lower continuum are occupied by electrons.

It turns out, however, that the presence of many electrons does not lead to any additional problems if the electron-electron interaction is neglected (or approximated by an effective singleparticle potential, Thomas-Fermi, HartreeFock, etc.). Under this approximation the electrons can influence each other through the Pauli exclusion principle only. But since the timedependent wave functions $\Phi_{i}^{(+)}$of two electrons initially in different states remain orthogonal throughout the collision, cf. Eq. (2.12), the Pauli principle has no effect on the excitation rates. ${ }^{40,41}$ If one is not interested in correlations between several particles or holes, the excitation rates are given by an incoherent summation of singleparticle transition probabilities. This remains true also if the electron-electron interaction is approximated by a mean screening potential common to all electrons.

The many-particle aspects are most conveniently described in the language of second quantization. Working in the Heisenberg picture we introduce a constant state vector $|F\rangle$ defined by the preparation of the collision system. The field operator $\hat{\Psi}(\vec{x}, t)$ which contains the dependence on time is expanded in a Fock decomposition

$$
\hat{\Psi}=\sum_{i<F} \hat{d}_{i}^{\dagger} \Phi_{i}^{(+)}+\sum_{i>F} \hat{b}_{i} \Phi_{i}^{(+)},
$$

where $\Phi_{i}^{(+)}$denotes a complete set of unquantized wave functions and $\hat{d}_{i}^{\dagger}$ and $\hat{b}_{i}$ are creation operators for holes and annihilation operators for particles, respectively. They are defined with respect to the "Fermi level" $F$, the boundary between initially occupied and empty states.

The operators $\hat{b}_{i}^{\dagger}, \hat{b}_{i}, \hat{d}_{i}^{\dagger}$, and $\hat{d}_{i}$ satisfy the usual fermionic anticommutation relations. Their action on the Heisenberg state $|F\rangle$ is

$\hat{d}_{i}|F\rangle=0$ for $i<F, \quad \hat{b}_{i}|F\rangle=0$ for $i>F$.

The equation of motion reads

$$
\frac{i \partial}{\partial t} \hat{b}_{i}=\sum_{k} \hat{b}_{k}\left\langle\Phi_{i}^{(+)}\left|H-i \frac{\partial}{\partial t}\right| \Phi_{k}^{(+)}\right\rangle,
$$

therefore, the $\hat{b}_{i}, \hat{d}_{i}$ become constant if the basis $\Phi_{i}^{(+)}$is identified with the set of solutions of the time-dependent single-particle Dirac equation (2.7). Since the basis $\Phi_{i}^{(+)}$satisfies the boundary condition at $t \rightarrow-\infty$ the number operators $\hat{d}_{i}^{\dagger} \hat{d}_{i}$, $\hat{b}_{i}^{\dagger} \hat{b}_{i}$ do not describe physically observable par- ticles after the collision. Instead, one has to employ a set which asymptotically correlates to a definite final state of the separated system. Using $\Phi_{i}^{(-)}$, the dynamic solutions of (2.1) satisfying outgoing boundary condition, one has the alternate expansion

$$
\hat{\Psi}=\sum_{i<F} \hat{d}_{i}^{\dagger} \Phi_{i}^{(-)}+\sum_{i>F} \hat{b}_{i} \Phi_{i}^{(-)} .
$$

Equating (2.17) and (2.20) yields a canonical transformation between the two sets of particle and hole operators, where the expansion coefficients $\left\langle\Phi_{j}^{(-)} \mid \Phi_{i}^{(+)}\right\rangle$are just the amplitudes $a_{i j}(\infty)$ discussed above

$$
\begin{aligned}
& \underline{\hat{d}}_{i}^{\dagger}=\sum_{k<F} \hat{d}_{k}^{\dagger} a_{k i}+\sum_{k>F} \hat{b}_{k} a_{k i} \text { for } i<F \\
& \underline{\hat{b}}_{i}=\sum_{k<F} \hat{d}_{k}^{\dagger} a_{k i}+\sum_{k>F} \hat{b}_{k} a_{k i} \text { for } i>F .
\end{aligned}
$$

The number of particles created in a state above the Fermi level $i>F$ is

$$
N_{i}=\left\langle F\left|\underline{\hat{b}}_{i}^{\dagger} \underline{\hat{b}}_{i}\right| F\right\rangle=\sum_{k<F}\left|a_{k i}\right|^{2}
$$

and the number of holes in a state below the Fermi level $i<F$ is

$$
\bar{N}_{i}=\left\langle F\left|\underline{\hat{d}}_{i}^{\dagger} \hat{\hat{d}}_{i}\right| F\right\rangle=\sum_{k>F}\left|a_{k i}\right|^{2} .
$$

These simple results contain a summation over all possible many-electron configurations with a particle (hole) in the level $i$. If one is interested in more detailed information on the final-state additional coherent terms arise. ${ }^{41,42}$ The number of correlated particle-hole pairs is given by the expectation value of the product of number operators

$$
\begin{aligned}
N_{i j} & =\left\langle F\left|\hat{b}_{i}^{\dagger} \hat{\underline{b}}_{i} \underline{\hat{d}}_{j}^{\dagger} \underline{\hat{d}}_{j}\right| F\right\rangle \\
& =N_{i} \bar{N}_{j}+\left|\sum_{k>F} a_{k i}^{*} a_{k j}\right|^{2} .
\end{aligned}
$$

The same formula holds also in the case of particle-particle and hole-hole correlations if the plus sign is replaced by a minus sign. The first term of (2.24) describes statistical coincidences while the sum contains coherent correlation effects. When the excitation rates are low, the second term becomes dominant since the probability for multiple excitations decreases rapidly. To analyze experiments which do not distinguish between several states (e.g., spin degeneracy) additional incoherent terms have to be added. ${ }^{43}$ Electron-positron coincidences are discussed in more detail in the Appendix. 


\section{DYNAMICAL TREATMENT OF SUPERCRITICAL COLLISIONS}

The theoretical discussion up to now has been limited to subcritical collisons where the energy eigenvalues of the adiabatic electronic bound states are confined to the gap region $-m c^{2}<E<m c^{2}$. In supercritical collisions, the deepest bound state joins the lower continuum and becomes a resonance. In the static limit, a hole brought into this state will decay spontaneously by positron emission leaving a stable filled atomic $K$ shell. ${ }^{1}$ The lifetime of the resonance is of the order $10^{-19} \mathrm{~s}$ and therefore considerably larger than the collision time $\left(\simeq 2 \times 10^{-21} \mathrm{~s}\right.$ for $\mathrm{U}-\mathrm{U}$ collisions with $r<R_{\text {cr }}$ ). Excitations induced by the nuclear motion will be of eminent importance. Therefore a formalism is required which describes dynamical excitations and at the same time accounts for the resonance character of the supercritical state.

The coupled differential equations (2.7) are not directly applicable to this situation: In the region $|t|<t_{\text {cr }}$ the $1 s \sigma$ state together with its amplitude disappears from the set of discrete states. Instead, the radial coupling matrix elements involving the lower continuum develop very strong and narrow (few keV) maxima near the (timedependent) position of the resonance. These couplings are not suitable for numerical treatment. In the following we will briefly discuss several possible methods of treating excitations involving the time-dependent resonance and illustrate the difficulties encountered. A projection method which seems to be best suited for practical calculations will be introduced and developed in detail in the next section.

(i) The static approximation. Here one assumes an undisturbed decay of the resonance taking place at each point of the trajectory. The transition rate is proportional to the decay width $\Gamma(t)$ which is determined parametrically by the nuclear motion. ${ }^{44,45}$ This approximation is insufficient; it does not take into account the finite oscillations of the phase factor in (2.7). Coherence of hole excitation and positron emission and, most important, the consequences of dynamical broadening may not be neglected.

(ii) Discretization of the continuum. When using the adiabatic basis in the supercritical case the $1 s \sigma$ state is represented by a narrow resonance in the negative energy continuum. In any numerical calculation with a reasonable mesh size the resonance position will only accidentally coincide with a grid point making a straightforward solution of the coupled-channel equations impossible. To ensure the inclusion of the resonance state at any internuclear distance $R$, the spec- trum of the Dirac Hamiltonian could be discretized by imposing a boundary condition on the wave functions at the surface of a sufficiently large volume. As sketched schematically in Fig. 1 , the $1 s \sigma$ level then joins the lower continuum as an additional state. ${ }^{6,7}$ Its wave function could be traced by a series of avoided crossings with very large radial coupling matrix elements. In any collision with nonvanishing velocity a $1 s \sigma$ hole will follow the "diabatic" state. Only a minute fraction of the number of holes will remain in the continuum. Obviously it will be very difficult to calculate this probability with any precision using the discretized adiabatic basis.

(iii) Use of a subcritical basis. Problems associated with the dynamical treatment of the resonance might be avoided by using a basis which remains subcritical throughout the whole collision. This is most easily achieved if one uses not the adiabatic eigenstates of $H(R(t))$, but those of a modified Hamiltonian $H(\rho(t))$, where $\rho(t)$ describes a trajectory satisfying $\rho>R_{\text {cr. }}$. The function $\rho(t)$ may be chosen arbitrarily, the simplest choice being $\rho(t)=R(t)$ for $|t|>t_{0}$ and $\rho(t)$ $=R_{0}$ for $|t|<t_{0}$. For $R_{0}=R_{\mathrm{cr}}$ this corresponds to a switching from the adiabatic basis in the subcritical region to a frozen basis in the supercritical domain.

If the colliding nuclei approach to distances $R(t)$ closer than $R_{0}$, there will arise potential couplings due to the operator $\Delta H=H(R(t))-H\left(R_{0}\right)$. The coupled-channel equations then are modified according to

$$
\begin{aligned}
\dot{a}_{i j}(t)=-\sum_{k \neq j} a_{i k}(t) & \\
& \times e^{-i\left(x_{k}-x_{j}\right)}\left\{\begin{array}{l}
\left\langle\phi_{j}|\partial / \partial t| \phi_{k}\right\rangle \\
\left\langle\phi_{j}^{0}|i \Delta H| \phi_{k}^{0}\right\rangle
\end{array} \text { for }|t|>t_{0}\right.
\end{aligned}
$$

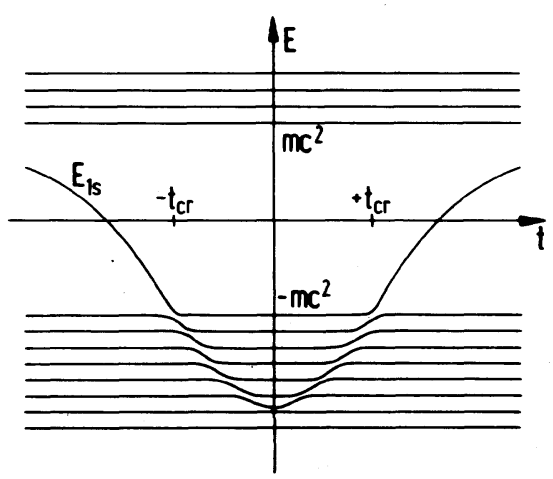

FIG. 1. Schematic graph of the 1s $\sigma$ energy as a function of time in a supercritical collision assuming a discretization of the spectrum. 
where $\phi_{k}^{0} \equiv \phi_{k}\left(R\left(t_{0}\right)\right)$ denotes the fixed basis states.

This description in principle is correct and the couplings are easily numerically manageable. It turns out, however, that it is very difficult to achieve completeness in the modified basis $\phi_{k}^{0}$. The nondiagonal matrix elements of $\Delta H$ decrease only very slowly with increasing energy separation between initial and final states so that a forbiddingly large number of channels would have to be included in the calculation. As an example, some radial and potential couplings from the $1 \mathrm{~s}$ state in the subcritical system $\mathrm{Pb}-\mathrm{Pb}$ are given in Table I. In the upper-half the completeness relation

$$
\left\langle\phi_{1 s}\left|\frac{\partial^{2}}{\partial R^{2}}\right| \Phi_{1 s}\right\rangle=-\sum_{k \neq 1 s}\left|\left\langle\phi_{k}\left|\frac{\partial}{\partial R}\right| \phi_{1 s}\right\rangle\right|^{2}
$$

is tested. The summation includes eight bound $s_{1 / 2}$ states (first line) and in addition the electron continuum up to $E_{e}=6 m c^{2}$ (second line). The wave functions have been calculated in monopole approximation, and the expectation value of $\partial / \partial R$ was obtained by numerical differentiation. In view of the limited numerical accuracy Eq. (3.2) is rather well fulfilled for the chosen basis. The situation is drastically different for the potential couplings (lower-half of Table I). Here the basis wave functions $\phi_{k}^{0}$ have been calculated for the fixed distance $R_{0}=50 \mathrm{fm}$, the matrix elements were evaluated at $R=16$ and $40 \mathrm{fm}$. In the comparison of $\left\langle\phi_{1 s}\left|(\Delta H)^{2}\right| \phi_{1 s}\right\rangle$ and $\sum_{k}\left|\left\langle\phi_{k}|\Delta H| \phi_{1 s}\right\rangle\right|^{2}$ the calculated value of the sum is much too small. This demonstrates that it is necessary to include electron states of very high energy if one tries to

TABLE I. Check of the completeness relation using the adiabatic basis (upper-half) and a basis frozen at $R_{0}=50 \mathrm{fm}$ (lower-half). The wave functions are calculated in monopole approximation for two internuclear distances. Eight bound $s_{1 / 2}$ states and continuum electrons up to $6 m c^{2}$ are included.

\begin{tabular}{ccc}
\hline \hline & $R=16 \mathrm{fm}$ & $R=40 \mathrm{fm}$ \\
\hline$\sum_{k \leq 8 s}\left|\left\langle\phi_{k}|\partial / \partial R| \phi_{1 s}\right\rangle\right|^{2}$ & 14.9 & 3.70 \\
$\mathcal{X}\left|\left\langle\phi_{k}|\partial / \partial R| \phi_{1 s}\right\rangle\right|^{2}$ & 27.3 & 7.23 \\
$-\left\langle\phi_{1 s}\left|\partial^{2} / \partial R^{2}\right| \phi_{1 s}\right\rangle$ & 32.2 & 8.34 \\
$\sum_{k \leq 8 s}\left|\left\langle\phi_{k}|\Delta H| \phi_{1 s}\right\rangle\right|^{2}$ & 0.027 & 0.004 \\
$\sum^{X}\left|\left\langle\phi_{k}|\Delta H| \phi_{1 s}\right\rangle\right|^{2}$ & 0.105 & 0.015 \\
$\left\langle\phi_{1 s}\left|(\Delta H)^{2}\right| \phi_{1 s}\right\rangle$ & 1.95 & 0.154 \\
\hline \hline
\end{tabular}

expand the time-dependent electronic wave function in a subcritical basis.

\section{A PROJECTION METHOD FOR THE SUPERCRITICAL RESONANCE STATE}

A. General considerations

The methods used to describe excitations in supercritical collisions discussed in the last section all had some serious disadvantages making them unsuitable for numerical calculations. Now we will develop a formalism ${ }^{26,46}$ which avoids these difficulties and moreover has heuristic value for the interpretation of the positron-creation process. We start from the observation that the continuum wave function of the supercritical system at resonance energy $E_{p}=E_{\text {res }}$ is quite similar to the discrete $1 \mathrm{~s} \sigma$ state in the subcritical case. In addition to a strongly localized density distribution having the extension of the atomic $K$ shell, the former exhibits an oscillating tail (small in amplitude) reaching out to infinity (see Fig. 2). This structure reflects the occurrence of a tunneling process through the gap separating the particle and antiparticle solutions of the Dirac equation (cf. the problem of Klein's paradox quoted in the introduction). Apart from the asymptotic behavior the $1 s \sigma$ wave function retains much of its identity. Many properties, e.g., the radial matrix elements for ionization, may be continued smoothly to the supercritical region just by neglecting the tail of the wave function. This procedure can be put on a firmer basis. In a first step a "quasibound" resonance state $\left|\Phi_{R}\right\rangle$ is defined as a reasonable

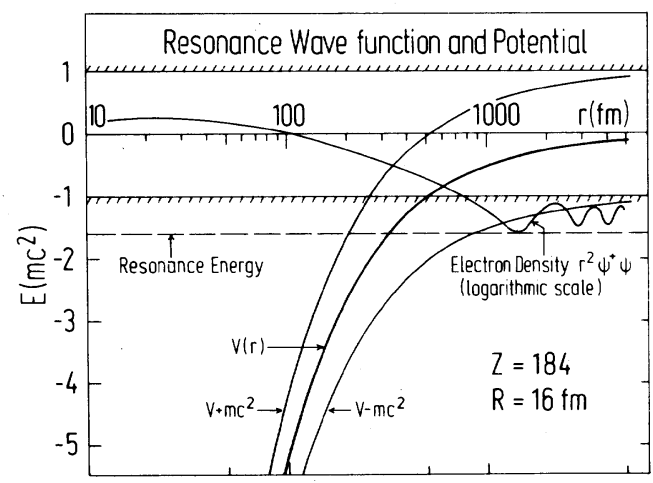

FIG. 2. The potential well $V(r)$ in a U-U quasimolecule near nuclear contact $(R=16 \mathrm{fm})$. Also shown are the borders of the gap $V(r) \pm m c^{2}$, the energy of the $1 s \sigma$ resonance, and the density of the positron continuum wave function at resonance energy. The density is drawn on a logarithmic scale covering ca. five orders of magnitude. 
approximation to the $1 s \sigma$ state in the absence of a penetrable tunneling barrier. In a second step a new positron continuum $\left|\tilde{\phi}_{E_{p}}\right\rangle$ has to be constructed which excludes the resonance, is orthogonal to $\left|\Phi_{R}\right\rangle$, and preserves completeness. This is achieved with a projection-operator technique which had been developed for applications in nuclear physics. ${ }^{47}$ The resulting set of modified stationary states will be used as a basis for expanding the time-dependent wave function in analogy with Eq. (2.5).

\section{B. The projection method}

We start from the assumption that a "physically reasonable" wave function $\left|\Phi_{R}\right\rangle$ describing the bound-state properties of the resonance has been found (for a further discussion see below). This state should be normalized

$$
\left\langle\Phi_{R} \mid \Phi_{R}\right\rangle=1
$$

and orthogonal to the states $\left|\phi_{\alpha}\right\rangle$ outside the positron continuum

$$
\left\langle\phi_{\alpha} \mid \Phi_{R}\right\rangle \simeq 0,
$$

where $\left|\phi_{\alpha}\right\rangle$ denotes all bound states and the electron continuum. The resonance now is to be extracted from the positron continuum $\left|\phi_{E_{p}}\right\rangle$ resulting in a modified continuum $\left|\tilde{\phi}_{E_{p}}\right\rangle$ orthogonal to the bound state

$$
\left\langle\tilde{\phi}_{E_{p}} \mid \Phi_{R}\right\rangle=0
$$

which still spans the same subspace.

This problem was solved in nuclear physics in connection with the continuum shell model. ${ }^{47-52}$ The applied formalism makes use of projection operators $P$ and $Q$, introduced by Feshbach, ${ }^{53}$ projecting on "open" and "closed" channels. In the subcritical case they will be defined to project on the space of continuum positron states

$$
P_{0}=\int d E_{p}\left|\phi_{E_{p}}\right\rangle\left\langle\phi_{E_{p}}\right|
$$

and on its complement

$$
\begin{aligned}
Q_{0} & =\sum_{n}\left|\phi_{n}\right\rangle\left\langle\phi_{n}\left|+\int d E_{e}\right| \phi_{E_{e}}\right\rangle\left\langle\phi_{E_{e}}\right| \\
& \left.\equiv \phi_{\alpha}\right\rangle\left\langle\phi_{\alpha}\right| .
\end{aligned}
$$

$\left|\phi_{E_{p}}\right\rangle$ and $\left|\phi_{\alpha}\right\rangle$ are eigenstates of the Hamiltonian $\boldsymbol{H}$,

$$
\left(E_{p}-H\right)\left|\phi_{E_{p}}\right\rangle=0 \text { and }\left(E_{\alpha}-H\right)\left|\phi_{\alpha}\right\rangle=0,
$$

so that $P_{0}$ and $Q_{0}$ satisfy the usual relations of orthogonal projection operators

$$
P_{0}^{2}=P_{0}, \quad Q_{0}^{2}=Q_{0}, \quad P_{0} Q_{0}=0 \text { ： }
$$

If the strength of the potential exceeds the cri- tical value the $1 s \sigma$ state becomes a resonance in the positron continuum thus entering $P_{0}$ space. The aim now is to transfer the bound-state contribution represented by the wave function $\Phi_{R}$ to $Q$ space. We define the new projection operators

$$
\begin{aligned}
& Q=\boldsymbol{y}\left|\phi_{\alpha}\right\rangle\left\langle\phi_{\alpha}|+| \Phi_{R}\right\rangle\left\langle\Phi_{R}\right|, \\
& P=1-Q:=\int d E_{p}\left|\tilde{\phi}_{E_{p}}\right\rangle\left\langle\tilde{\phi}_{E_{p}}\right| .
\end{aligned}
$$

Under the assumption (4.2) the operators $Q ; P$ again are orthogonal projectors. If $\Phi_{R}$ was chosen judiciously the newly defined modified continuum $\bar{\phi}_{E_{\phi}}$ will no longer show resonance behavior. The $\left|\vec{\phi}_{E_{p}}\right\rangle$ are eigenstates of the Hamiltonian restricted to the subspace $P$ (Ref. 47 ), i.e.,

$$
\left(E_{p}-P H P\right)\left|\tilde{\phi}_{E_{p}}\right\rangle=0 \text {. }
$$

Using (4.6), (4.7), and the orthogonality relations (4.2) and (4.3), this equation may be transformed to a more explicit form

$$
\left(E_{p}-H\right)\left|\tilde{\phi}_{E_{p}}\right\rangle=-\left\langle\Phi_{R}|H| \tilde{\phi}_{E_{p}}\right\rangle\left|\Phi_{R}\right\rangle .
$$

The modified continuum states satisfy the original Dirac equation supplemented by an inhomogeneous term containing an integral over the solution $\tilde{\phi}_{E_{p}}$. Fortunately the kernel is separable so that $(4.9)$ can be solved easily.

The formal solution of Eq. (4.9) using the Green's function $G$ with $(E-H) G=1$ has been given in Refs. 47 and 52. Projecting the general solution

$$
\left|\tilde{\phi}_{E_{p}}\right\rangle=C\left|\phi_{E_{p}}\right\rangle-\left\langle\Phi_{R}|H| \tilde{\phi}_{E_{p}}\right\rangle G\left|\Phi_{R}\right\rangle
$$

on $\left\langle\Phi_{R}\right|$ and imposing the orthogonality relation (4.3) we obtain

$$
\left|\tilde{\phi}_{E_{p}}\right\rangle=C\left(1-\frac{G\left|\Phi_{R}\right\rangle\left\langle\Phi_{R}\right|}{\left\langle\Phi_{R}|G| \Phi_{R}\right\rangle}\right)\left|\phi_{E_{p}}\right\rangle .
$$

$C$ is a normalization constant which depends on the boundary conditions and has absolute value of unity if the propagator for outgoing waves is used. This is further discussed in Ref. 52 .

Using the solution (4.11) it can be shown that

$$
\left\langle\tilde{\phi}_{B_{p}^{\prime}} \mid \tilde{\phi}_{E_{p}}\right\rangle=\delta\left(E_{p}^{\prime}-E_{p}\right)
$$

and also

$$
\left\langle\tilde{\phi}_{E_{p}^{\prime}}^{\prime}|H| \tilde{\phi}_{E_{p}}\right\rangle=E \delta\left(E_{p}^{\prime}-E_{p}\right) .
$$

The modified continuum satisfies the same orthonormality relations as the old one. Its phase shift, however, is changed by a counter term which cancels the steep variation near the position of the resonance and leaves a smooth nonresonating phase. ${ }^{47}$ 
Equation (4.11) might be solved with the use of the Green's function $G$ represented in coordinate space. For a spherically symmetric potential (monopole approximation) with asymptotic $1 / r$ behavior $G\left(r, r^{\prime}\right)$ expressed in terms of regular and irregular solutions of the radial equation is well known. ${ }^{54}$ On the other hand, the defining equation (4.9) can be solved directly by a straightforward integration procedure as reported by Wang and Shakin. ${ }^{47}$ It consists of the following steps:

(i) Choose two different arbitrary constants $\gamma^{(1)}$ and $\gamma^{(2)}$.

(ii) Solve the inhomogenous Dirac equations for each constant

$$
(H-E)\left|\phi_{E}^{i}\right\rangle=\gamma^{(i)}\left|\Phi_{R}\right\rangle \text {. }
$$

(iii) Represent the general solution by a linear combination

$$
\left|\tilde{\phi}_{E}\right\rangle=a\left|\phi_{E}^{(1)}\right\rangle+b\left|\phi_{E}^{(2)}\right\rangle \text {. }
$$

(iv) The orthogonality requirement $\left\langle\Phi_{R} \mid \tilde{\phi}_{E}\right\rangle=0$ eliminates one of the constants

$$
\left|\tilde{\phi}_{E}\right\rangle=a\left(\left|\phi_{E}^{(1)}\right\rangle-\frac{\left\langle\Phi_{R} \mid \phi_{E}^{(1)}\right\rangle}{\left\langle\Phi_{R} \mid \phi_{E}^{(2)}\right\rangle}\left|\phi_{E}^{(2)}\right\rangle\right) \text {. }
$$

(v) The wave function is normalized asymptotically $(r-\infty)$.

The resulting $\tilde{\phi}_{E}$ is independent of the choice of the constants $\gamma^{(i)}$. The nondiagonal matrix elements of the Hamiltonian follow as

$$
\left\langle\Phi_{R}|H| \tilde{\phi}_{E}\right\rangle=a\left(\gamma^{(1)}-\frac{\left\langle\Phi_{R} \mid \phi_{E}^{1}\right\rangle}{\left\langle\Phi_{R} \mid \phi_{E}^{2}\right\rangle} \gamma^{(2)}\right) \text {. }
$$

The projection formalism is easily extended to the case of several resonances. In the present context this is not required since in heavy-ion collisions apart from the $1 s \sigma$ only the $2 p_{1 / 2} \sigma$ state becomes supercritical (at $Z \sim 185$ ). In symmetric systems and generally if the monopole approximation is used the $p_{1 / 2} \sigma$ and $s_{1 / 2} \sigma$ continua do not couple and can be treated independently. The $2 s \sigma$ state dives at $Z \sim 245$, which is far too high to be reached in a collision of two heavy ions. For completeness we will quote the results ${ }^{52}$ applicable to the case of several resonances.

We assume that $N$ resonances have to be extracted from the continuum for which a suitable set of resonance states $\left|\Phi_{i}\right\rangle$ has been defined, satisfying

$$
\left\langle\Phi_{i} \mid \Phi_{j}\right\rangle=\delta_{i j},\left\langle\phi_{\alpha} \mid \Phi_{i}\right\rangle \simeq 0 .
$$

The projection operators now are

$$
\begin{aligned}
& Q=\boldsymbol{X}\left|\phi_{\alpha}\right\rangle\left\langle\phi_{\alpha}\left|+\sum_{i=1}^{N}\right| \Phi_{i}\right\rangle\left\langle\Phi_{i}\right|, \\
& P=1-Q,
\end{aligned}
$$

and the inhomogeneous Dirac equation (4.9) is generalized to

$$
(E-H)\left|\tilde{\phi}_{E}\right\rangle=-\sum_{i=1}^{N}\left|\Phi_{i}\right\rangle\left\langle\Phi_{i}|H| \tilde{\phi}_{E}\right\rangle
$$

which still is separable. The formal solution of (4.18) is

$$
\left|\tilde{\phi}_{E}\right\rangle=C\left(1-G \sum_{i, j=1}^{N}\left|\Phi_{i}\right\rangle\left\langle\left\langle\Phi_{k}|G| \Phi_{1}\right)_{i j}^{-1}\left\langle\Phi_{j}\right|\right)\left|\phi_{E}\right\rangle,\right.
$$

where the matrix $\left\langle\Phi_{k}|G| \Phi_{1}\right\rangle$ has to be inverted. Similarly, the direct integration method can be extended ${ }^{55}$ to include several resonances. Setting $\gamma^{(1)}=0$ the inhomogeneous differential equations

$$
(H-E)\left|\phi_{E, i}^{(2)}\right\rangle=\gamma^{(2)}\left|\Phi_{i}\right\rangle .
$$

have to be solved for all $i=1, \ldots, N$. The ansatz

$$
\left|\tilde{\phi}_{E}\right\rangle=a\left|\phi_{E}\right\rangle+\sum_{i=1}^{N} b_{i}\left|\phi_{E, i}^{(2)}\right\rangle
$$

together with the orthogonality requirement (4.3) leads to a system of $N$ linear equations. The resulting modified continuum state is

$$
\left|\tilde{\phi}_{E}\right\rangle=a\left(\left|\phi_{E}\right\rangle-\sum_{i, j=1}^{N}\left(\left\langle\Phi_{k} \mid \phi_{E, 1}^{(2)}\right\rangle\right)_{i j}^{-1}\left\langle\Phi_{j} \mid \phi_{E}\right\rangle\left|\phi_{E, i}^{(2)}\right\rangle\right) \text {. }
$$

The resonance state $\left|\Phi_{R}\right\rangle$ and its associated modified positron continuum $\left|\tilde{\phi}_{E}\right\rangle$ have to be determined at all values of the internuclear distance $R<R_{\text {cr }}$. They will be used as a basis to expand the time-dependent wave function in complete analogy with the subcritical case (2.5), i.e.,

$$
\begin{aligned}
\Phi_{i}(t)= & \int_{m}^{\infty} d E_{e} a_{i, E_{e}} \phi_{E_{e}} e^{-i E_{e} t}+\sum_{n>1 s} a_{i, n} \phi_{n} e^{-i X_{n}} \\
& +a_{i, 1 s} \Phi_{R} e^{-i X_{1 s}}+\int_{-\infty}^{-m} d E_{p} a_{i, E_{p}} \tilde{\phi}_{E_{p}} e^{-i E_{p} t} .
\end{aligned}
$$

If the resonance energy $E_{1 s}$ is identified with $\left\langle\Phi_{R}|H| \Phi_{R}\right\rangle$ and the or thogonality relation (4.2) is fulfilled, we obtain the old set of coupled differential equations (2.7). There arises, however, one important modification: Since the resonance state $\left|\Phi_{R}\right\rangle$ is not an eigenstate of $H$ it has an additional interaction with the continuum. The coupling matrix elements $1 s \sigma \rightarrow E_{p}$ must be replaced by

$\left\langle\phi_{E_{p}}\left|\frac{\partial}{\partial t}\right| \phi_{1 s}\right\rangle-\left\langle\tilde{\phi}_{E_{p}}\left|\frac{\partial}{\partial t}\right| \Phi_{R}\right\rangle+i\left\langle\tilde{\phi}_{E_{p}}|H| \Phi_{R}\right\rangle$.

A hole prepared in $\Phi_{R}$ therefore will decay by spontaneous positron emission in addition to the dynamically induced transitions described by the $\partial / \partial t$ coupling. In the static limit, $R(t)=$ const, this leads to an exponential decay with the width 


$$
\Gamma=2 \pi\left|\left\langle\tilde{\phi}_{E_{p}}|H| \Phi_{R}\right\rangle\right|^{2},
$$

where $E_{p}$ is taken at the position of the resonance. The developed formalism thus has led to the emergence of "induced" and "spontaneous" positron couplings, the latter resulting from the presence of an unstable state $\Phi_{R}$ in the expansion basis. It is difficult, however, to draw simple conclusions from this fact. Both coupling matrix elements enter via their Fourier transforms, depending on the time development of the heavyion collision. Their contributions have to be added coherently so that in a given collision there is no physical way to distinguish between them. As discussed in Sec. V, significant deviations of the positron-production rate in supercritical collision systems are expected only under favorable conditions, i.e., in encounters with a prolonged interaction time.

\section{Wave functions and matrix elements}

The projection method for constructing a modified basis described in the last section starts from the quasibound state $\left|\Phi_{R}\right\rangle$. We have to find a prescription which generates a wave function $\left|\Phi_{R}\right\rangle$ with the properties of a $1 s \sigma$ state. In particular, its binding energy and the radial coupling matrix elements to higher $s$-like bound and continuum states should increase in smooth continuation of the values in the subcritical region $R>R_{\mathrm{cr}}$.

The construction of $\left|\Phi_{R}\right\rangle$, in principle, is quite arbitrary. In a natural way this may be accomplished by defining a resonance wave packet as a superposition of the old continuum states $\phi_{E_{p}}$, integrated over a suitable energy interval which contains the position of the resonance. Such a definition was employed in Ref. 17 to study the spontaneous decay of the $1 s$-hole state. In Ref. 6 the wave packet was used to obtain the density distribution of the supercritical $K$ shell. The practical construction of $\left|\Phi_{R}\right\rangle$, however, seems to be quite tedious, if one wants to avoid further approximations. In the following therefore we will use another definition.

The most straightforward prescription is to start from the positron continuum wave function $\phi_{E_{p}}$ at the energy of the resonance $E_{p}=E_{r o s}$, cutting it off at large distances $r>_{r_{c}}$ :

$$
\Phi_{R}(r)=C \phi_{E_{\text {res }}}(r) \Theta\left(r_{c}-r\right) .
$$

The normalization constant $C$ is determined by

$$
C=\left(\int_{0}^{r_{c}} r^{2} d r \int d \Omega \phi_{E_{\mathrm{ros}}}^{+} \phi_{E_{\mathrm{ros}}}\right)^{-1 / 2}
$$

so that (4.1) is fulfilled. The cut-off wave function (4.27) has been employed by Wang and Shakin ${ }^{47}$ and other $\mathrm{s}^{48-52}$ to describe resonances in nuclear physics. The cut-off distance $r_{c}$ is a somewhat arbitrary parameter which should be chosen to lie within the region of the tunneling barrier defined by $r_{-}<r<r_{+}$with $E_{\text {ros }}-V\left(r_{ \pm}\right) \pm m c^{2}=0$. At larger distances $r$ the wavefunction $\phi_{E_{p}}$ begins to oscillate. This is illustrated in Fig. 2 where the density of the positron wave function at $E=E_{\text {ros }}$ is shown together with the gap of the Dirac equation and the nuclear Coulomb potential $V(r)$. The system is $\mathrm{U}-\mathrm{U}$ at internuclear distance $R=16 \mathrm{fm}$, i.e., at nuclear contact.

To avoid problems associated with the discontinuity of the wave function introduced by (4.27) we have adopted a modified cut-off procedure for the following calculations: $\Phi_{R}$ will be defined as an eigenfunction of the Dirac Hamiltonian with the modified potential

$$
\tilde{V}(r)=\Theta\left(r_{c}-r\right) V(r)+\Theta\left(r-r_{c}\right) V\left(r_{c}\right) .
$$

For distances $r<r_{c}, \tilde{V}$ agrees with the old potential $V$; at large distances the potential is kept fixed so that the $1 s$ energy remains inside the gap region. This artifice produces a smoothly decreasing tail of $\Phi_{R}$, while the wave function agrees with $\phi_{E_{\text {res }}}$ in the interior region.

The value of $r_{c}$ is defined by $E_{R}-V\left(r_{c}\right)=-\gamma m c^{2}$, and we will use $\gamma=0.9$ in the following calculations. With this prescription $r_{c}$ is close to the outer turning point $r_{+}$. As an illustration, Fig. 2 shows the potential $V(r)$ and the resonance wave function obtained by this procedure for the system U-U at $R=16 \mathrm{fm}$. Since $r_{c}$ becomes large for $E_{R}$ close to the boundary of the continuum there is a smooth transition from the subcritical region. The distance $r_{c}$ is energy dependent, therefore the binding energy $E_{R}$ and the wave function $\Phi_{R}$ have to be obtained from a self-consistent solution of the Dirac equation

$$
\left(\tilde{H}-E_{R}\right)\left|\Phi_{R}\right\rangle=0 \text {, }
$$

where $\bar{H}=T+\tilde{V}$. As demonstrated in Table II, the value of $E_{R}$ agrees closely with the exact resonance energy obtained from a phase-shift analysis of the continuum wave function $\phi_{E_{p}}$. For the potential $V(r)$ we have taken the monopole part of the two-center potential assuming homogeneously charged extended nuclei ${ }^{56}$ with radius $r_{k}=1.2 A^{1 / 3} \mathrm{fm}$. Owing to the high localization of the wave function the effect of the finite nuclear extension is not negligible at the close internuclear distances considered here. For instance, the $1 s$ energy and width are $E_{R}=-1.8533, \Gamma=5.3$ $\mathrm{keV}$ for $\mathrm{U}-\mathrm{U}$, and $E_{R}=-2.3597, \Gamma=14.6 \mathrm{keV}$ for $\mathrm{U}-\mathrm{Cf}$ at $R=16 \mathrm{fm}$ for two-point nuclei. Obviously, the decrease of binding energies leads to a substantial reduction of the decay width.

Using the smoothly cut-off resonance wave func- 
TABLE II. Energy and width of the resonance in the $s_{1 / 2}$ positron continuum of the quasimolecules $U-U$ and U-Cf, calculated in monopole approximation for extended nuclei. $E_{\text {res }}$ and $\Gamma$ are determined by a phase-shift analysis of the continuum $\phi_{E}, E_{R}$ and $\Gamma_{R}$ are defined by Eqs. (4.30) and (4.26) using the truncated potential (4.29).

\begin{tabular}{llllll}
\hline \hline System & $R(\mathrm{fm})$ & $E_{\mathrm{res}}\left(m c^{2}\right)$ & $\Gamma(\mathrm{keV})$ & $E_{R}\left(m c^{2}\right)$ & $\Gamma_{R}(\mathrm{keV})$ \\
\hline \multirow{4}{*}{$\mathrm{U}+\mathrm{U}$} & 16 & -1.5894 & 2.00 & -1.5906 & 1.97 \\
& 20 & -1.3963 & 0.505 & -1.3967 & 0.503 \\
& 25 & -1.20840 & 0.023 & -1.20844 & 0.023 \\
& 30 & -1.06339 & $1(-6)$ & -1.06340 & $1.2(-6)$ \\
& 16 & -2.016 & 8.19 & -2.011 & 8.19 \\
$\mathrm{U}+\mathrm{Cf}$ & 20 & -1.7630 & 4.12 & -1.761 & 4.10 \\
& 25 & -1.5208 & 1.32 & -1.5199 & 1.31 \\
& 30 & -1.3367 & 0.245 & -1.3369 & 0.244 \\
\hline \hline
\end{tabular}

tion $\Phi_{R}$ defined by (4.30) with potential (4.29) we have constructed the modified continuum $\tilde{\phi}_{E}$. We have numerically integrated the inhomogeneous Dirac equation (4.14) with $\gamma^{(1)}=0$ and $\gamma^{(2)}=1$ (the result is independent of this special choice) and obtained $\tilde{\phi}_{E_{p}}$ from the superposition (4.15). The amplitude of the wave function was normalized to the analytic solution ${ }^{57}$ at $r=5000 \mathrm{fm}$. As an example, Fig. 3 shows the large and small components of $\phi_{E_{p}}$ at resonance energy and the cut-off wave function $\Phi_{R}$ (dotted lines). The lower part of the figure displays the modified continuum $\bar{\phi}_{E_{p}}$ which shows no resonance behavior. When studying the properties of the modified continuum it is

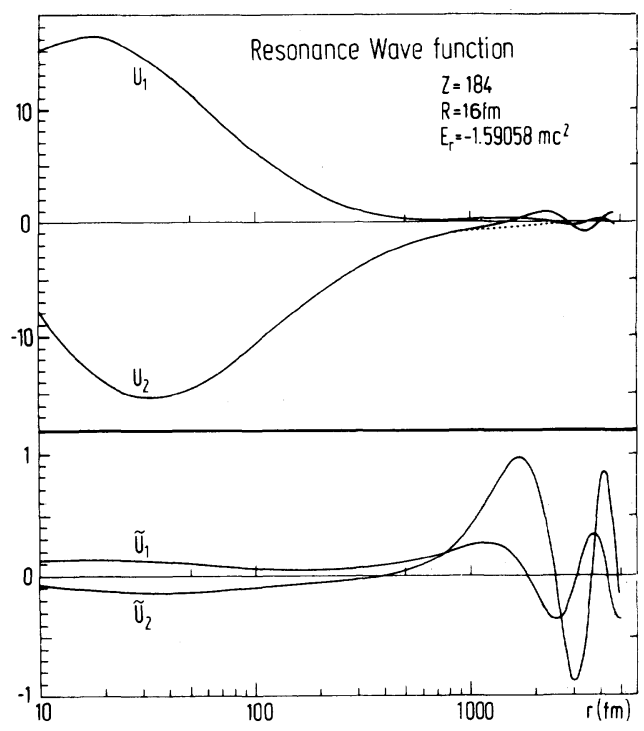

FIG. 3. Upper part: The large and small component $u_{1}$ and $u_{2}$ of the $s_{1 / 2}$ continuum as in Fig. 2. The dashed lines indicate the resonance wave function $\Phi_{R}$ (not normalized) as defined by Eq. (4.30). Lower part: The modified continuum $\tilde{\phi}_{E_{\mathrm{res}}}$. interesting to compare the phase shifts of $\phi_{E_{p}}$ and $\bar{\phi}_{E_{p}}$ in the vicinity of the resonance. For the $U-U$ system at $R=16 \mathrm{fm}$, Fig. 4 demonstrates the absence of any structure in the phase shift $\bar{\delta}$, while $\delta$ sharply increases by $\pi$ at $E=E_{\text {res: }}$. Thus the resonance has been completely eliminated from the continuum. Also shown in Fig. 4 is the "resonance excess" of the wave function $\phi_{E_{p}}$ defined as the ratio between the maximum and asymptotic value of $u_{1}(r)$, the large component of the radial wave function. The narrow BreitWigner-like maximum of this ratio characterizes a sharp resonance, its high value is support for the concept of defining a quasibound state by cutting off $\phi_{E_{\text {res }}}$.

Particularly important are the results for the decay width $\Gamma$ of the state $\Phi_{R}$ as expressed by the squared nondiagonal matrix element of the Hamiltonian at energy $E=E_{\text {res}}$, cf. Eq. (4.26). The values of $\Gamma$ were found to be largely independent of the details of the cut-off procedure for $\Phi_{R}$. Figure 5 shows the curves $\Gamma(R)$ for the systems $\mathrm{U}-\mathrm{U}(Z=184)$ and U-Cf $(Z=190)$. Note the strong decrease of $\Gamma$ with growing two-center distance and the high- $Z$ dependence. This implies that it will be necessary to study close collisions of very heavy systems if any effects of the spontaneous decay of the resonance are to be observed.

The projection method is substantially supported by the results of a direct phase-shift analysis of the continuum $\phi_{E_{p}}$. Table II gives the values of $\Gamma$ for several internuclear distances $R$. The

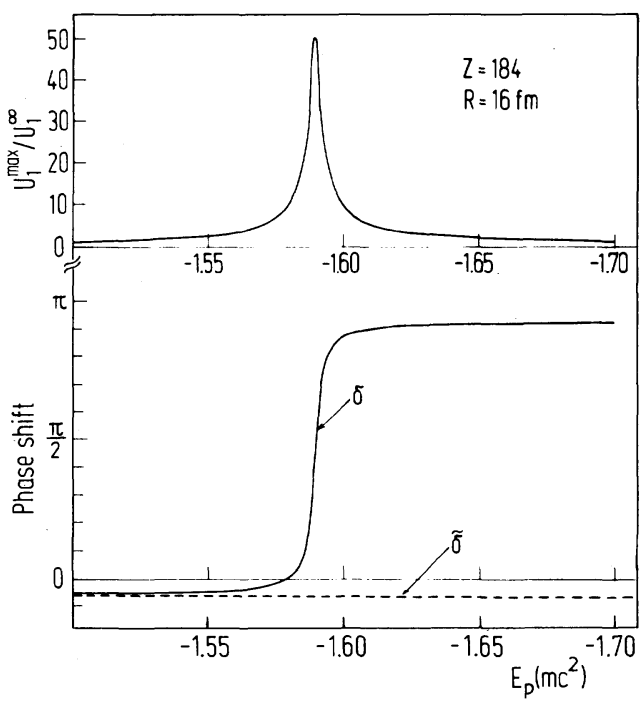

FIG. 4. Analysis of the resonance in the negative energy $s_{1 / 2}$ continuum in the U-U quasimolecule at $R$ $=16 \mathrm{fm}$. Upper part: The ratio of the maximum and asymptotic value of $u_{1}$. Lower part: The phase shift of $\phi_{E_{p}}$ and $\tilde{\phi}_{E_{p}}$, the original and modified continuum. 


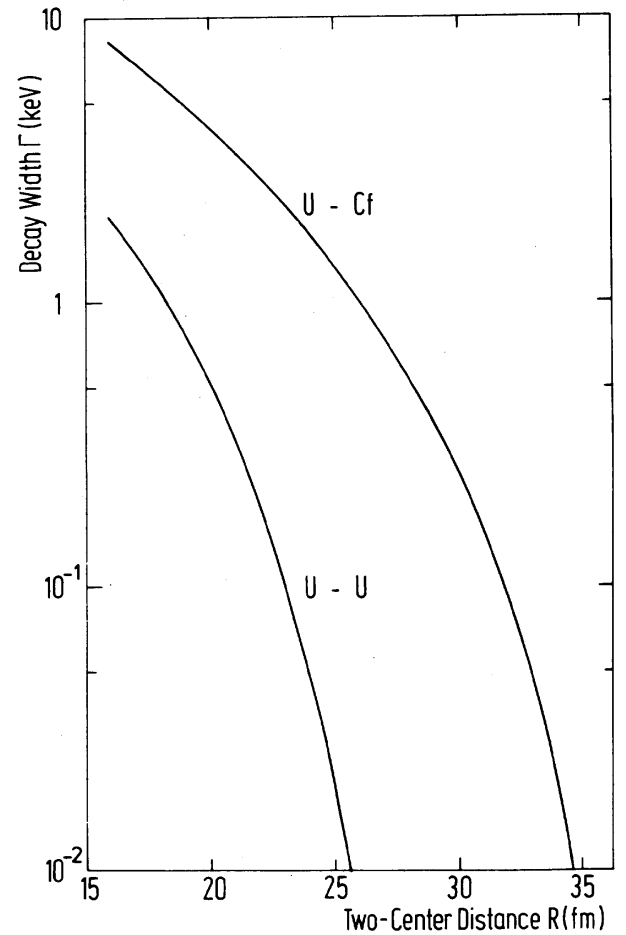

FIG. 5. Decay width $\Gamma$ in $\mathrm{keV}$ of the $1 s \sigma$ resonance in the system $\mathrm{U}-\mathrm{U}$ and $\mathrm{U}-\mathrm{Cf}$ as a function of internuclear distance $R$, calculated in monopole approximation.

widths obtained from the two methods are in very good agreement. Since the decay width $\Gamma$ $=2 \pi\left|\left\langle\tilde{\phi}_{E_{\text {res }}}|H| \Phi_{R}\right\rangle\right|^{2}$ provides an adequate description of the positron emission process only in the limit of infinite collision time we have to study the properties of the coupling matrix elements $\left\langle\tilde{\phi}_{E_{p}}|H| \Phi_{R}\right\rangle$ and $\left\langle\tilde{\phi}_{E_{p}}|\partial / \partial R| \Phi_{R}\right\rangle$ in more detail. Figure 6 shows the decay matrix element as a function of positron energy for the systems $U-U$ and U-Cf. The increase with two-center distance $R$, taken as a fixed parameter for each curve, has been noted already in the special case of the width. Aside from the Coulomb repulsion effect at small kinetic positron energies the curves show a broad maximum. The values at resonance energy $\left(E_{p}=E_{\text {res }}\right)$ are well defined. The detailed behavior of the energy dependence, however, in particular, the change of sign at high energy, depends rather sensitively on the employed cutoff procedure for $\Phi_{R}$.

The same is true for the radial coupling $\left\langle\tilde{\phi}_{E_{p}}|\partial / \partial R| \Phi_{R}\right\rangle$ between resonance state and positron continuum. The variation of the matrix elements with two-center distance is shown in Figs. 7 (a) and 7(b) for the two systems under consideration. The matrix elements have been calculated by numerical differentiation of the wave function $\Phi_{R}$. In contrast to the subcritical situa-

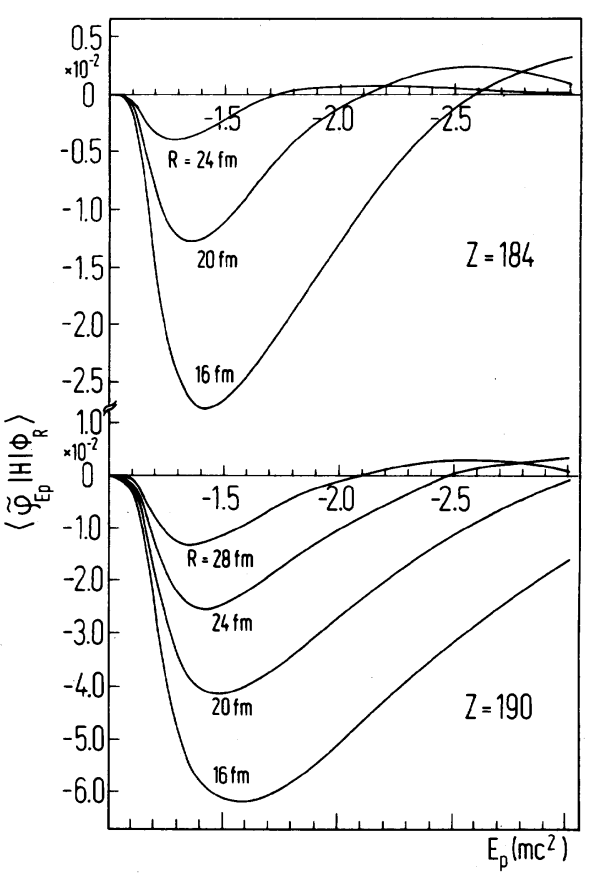

FIG. 6. The coupling matrix elements $\left\langle\tilde{\phi}_{E_{\rho}}|H| \Phi_{R}\right\rangle$ as a function of positron energy $E_{p}$ for the systems U-U and $\mathrm{U}-\mathrm{Cf}$.

tion the Hellmann-Feynman identity is not applicable directly since $\left|\tilde{\phi}_{E_{p}}\right\rangle$ and $\left|\Phi_{R}\right\rangle$ are not eigenstates of the same Hamiltonian.

The value of $\left\langle\tilde{\phi}_{E_{p}}|\partial / \partial R| \Phi_{R}\right\rangle$ joins smoothly with $\left\langle\phi_{E_{p}}|\partial / \partial R| \Phi_{R}\right\rangle$ at the critical distance $R=R_{\mathrm{cr}}$. This had been postulated at the outset as a criterion for the suitability of the projection method. While the matrix elements increase monotonically in the subcritical region, they reach a maximum and fall off again at small internuclear distance $R$. The position of the maximum shifts to smaller $R$ as the positron kinetic energy increases. It seems to be correlated, but not identical, with the distance where the resonance energy crosses the energy of the positron state under consideration. Except for the matrix elements joining $\Phi_{R}$, all further couplings to the modified continuum $\bar{\phi}_{E_{p}}$ show no structure, again demonstrating that the resonance has been successfully extracted from the continuum. To perform calculations of positron creation, knowledge of the radial coupling from $\tilde{\phi}_{E_{p}}$ to higher bound and continuum states is required. In calculating the matrix elements $\left\langle\phi_{\alpha}|\partial / \partial R| \Phi_{R}\right\rangle$ and $\left\langle\tilde{\phi}_{E_{p}}|\partial / \partial R| \Phi_{R}\right\rangle$, numerical differentiation of the resonance wave function was required. The numerical differentiation of $\tilde{\phi}_{E}$ can be avoided by using a modified form of the Hellmann-Feynman identity. We take the matrix element of the commutator $[\theta / \theta R, H]$ 

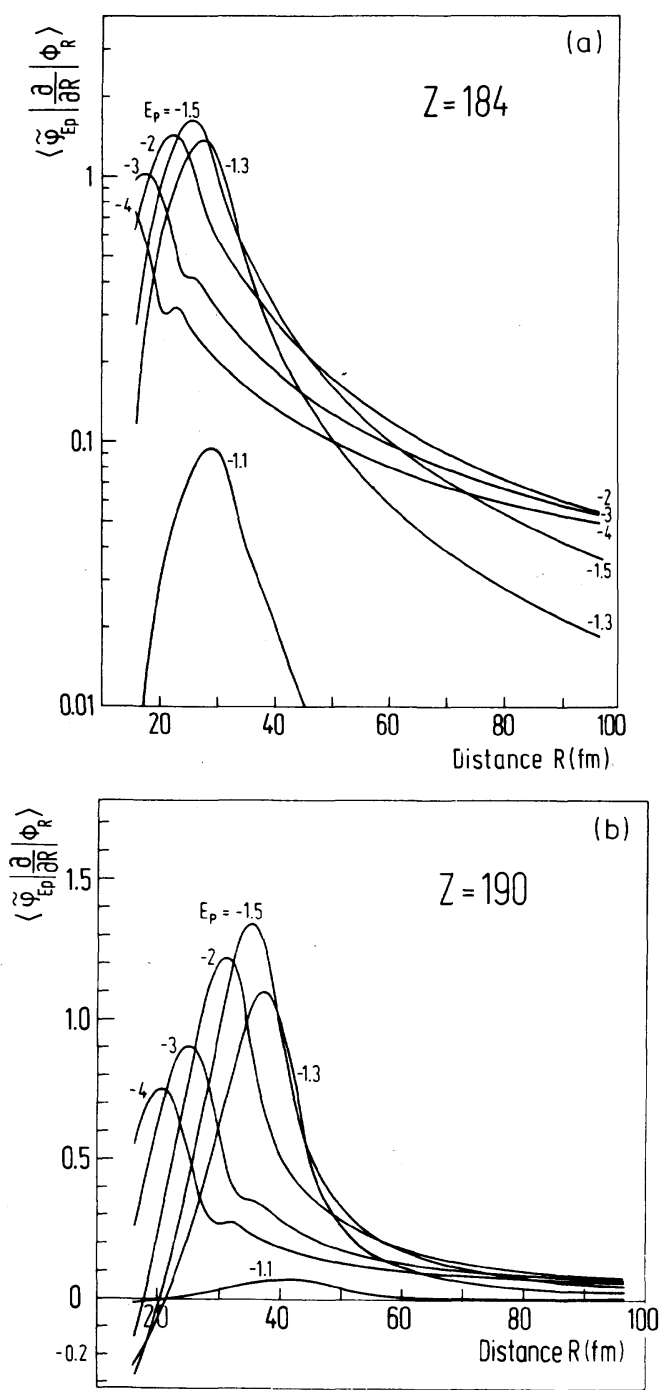

FIG. 7. The radial coupling matrix elements $\left\langle\tilde{\phi}_{E_{\phi}}|\partial / \partial R| \Phi_{R}\right\rangle$ as a function of internuclear distance $R$ for the systems U-U [part (a)] and U-Cf [part (b)].

$=\partial H / \partial R$ between the states $\left\langle\phi_{\alpha}\right|$ and $\left|\tilde{\phi}_{E_{p}}\right\rangle$. Use of (4.9) leads to

$$
\begin{aligned}
\left\langle\phi_{\alpha}\left|\frac{\partial}{\partial R}\right| \tilde{\phi}_{E_{p}}\right\rangle=\frac{1}{E_{p}-E_{\alpha}} & \left(\left\langle\phi_{\alpha}\left|\frac{\partial H}{\partial R}\right| \tilde{\phi}_{E_{p}}\right\rangle\right. \\
& -V_{E_{p}}^{*}\left\langle\phi_{\alpha}\left|\frac{\partial}{\partial R}\right| \Phi_{R}\right\rangle \\
& \left.-\frac{\partial V_{E_{p}}^{*}}{\partial R}\left\langle\phi_{\alpha} \mid \Phi_{R}\right\rangle\right),
\end{aligned}
$$

with the abbreviation $V_{E_{p}}^{*}=\left\langle\Phi_{R}|H| \bar{\phi}_{E_{p}}\right\rangle$. In addition to the usual $\theta H / \partial R$ term this expression contains a correction proportional to the decay matrix element $V_{E_{p}}$ and a nonorthogonality modifica- tion. Numerically the first two terms on the rhs of (4.31) were found to be of the same order, while the overlap correction amounts to less than $10 \%$. It will be neglected in the following calculations. The radial coupling among the states of the positron continuum can be treated in a similar manner. The resulting identity reads

$$
\begin{aligned}
\left\langle\tilde{\phi}_{E_{p}}\left|\frac{\partial}{\partial R}\right| \phi_{E_{\dot{p}}^{\prime}}\right\rangle=\frac{1}{E_{p}^{\prime}-E_{p}} & \left(\left\langle\tilde{\phi}_{E_{p}}\left|\frac{\partial H}{\partial R}\right| \phi_{E_{\dot{p}}^{\prime}}\right\rangle\right. \\
& -V_{E_{\dot{p}}}^{*}\left\langle\tilde{\phi}_{E_{p}}\left|\frac{\partial}{\partial R}\right| \Phi_{R}\right\rangle \\
& \left.+\dot{V}_{E_{p}^{\prime}}\left\langle\Phi_{R}\left|\frac{\partial}{\partial R}\right| \tilde{\phi}_{E_{\dot{p}}}\right\rangle\right) .
\end{aligned}
$$

A certain problem of the presented projection formalism is the requirement of orthogonality between the resonance wave function $\Phi_{R}$ and the states $\phi_{\alpha},\left\langle\phi_{\alpha} \mid \Phi_{R}\right\rangle=0$. For an arbitrary choice of $\Phi_{R}$ this condition will not be satisfied exactly. When the operators $P$ and $Q$ of Eq. (4.7) are not orthogonal projectors, the subsequent derivations entail an approximation. The problem might be circumvented by orthogonalizing $\Phi_{R}$ to all states $\phi_{\alpha}$, e.g., by Schmidt's procedure. This is hardly practical, however, since the set $\phi_{\alpha}$ contains the continuum of electron states $\phi_{E_{e}}$. Alternatively the states $\phi_{\alpha}$ might be treated in the same way as the $\phi_{E}$ so that only $\Phi_{R}$ remains in $Q$ space. The higher states then will be modified to a set $\phi_{\alpha}$ satisfying the analog of the inhomogeneous Dirac equation (4.9). This would lead to additional nondiagonal couplings through the Hamiltonian $H$.

In the present work we will neglect all errors introduced by the nonorthogonality $\left\langle\phi_{\alpha} \mid \Phi_{R}\right\rangle$. For the resonance state $\Phi_{R}$ defined according to (4.29), the overlap to higher $n s \sigma$ bound states was found to be smaller than $1 \times 10^{-2}$ in the worst case (U-Cf at $R=16 \mathrm{fm})$. The sum $\left(\sum_{n}\left|\left\langle\phi_{n} \mid \Phi_{R}\right\rangle\right|^{2}\right.$ $\left.+\int d E_{e}\left|\left\langle\phi_{E_{e}} \mid \Phi_{R}\right\rangle\right|^{2}\right)^{1 / 2}$ did not exceed $2 \times 10^{-2}$. The corresponding overlap of the modified continuum states $\tilde{\phi}_{E_{p}}$ can be reduced to $\left\langle\phi_{\alpha} \mid \Phi_{R}\right\rangle$ using (4.9):

$$
\left\langle\phi_{\alpha} \mid \tilde{\phi}_{E_{p}}\right\rangle=\frac{\left\langle\Phi_{R}|H| \phi_{E_{p}}\right\rangle}{E_{\alpha}-E_{p}}\left\langle\phi_{\alpha} \mid \Phi_{R}\right\rangle .
$$

According to the orthogonality assumption the coupling matrix (2.10) will be assumed antiHermitian. Otherwise the variable overlap matrix had to be included in the coupled differential equations, see (2.8).

\section{NUMERICAL RESULTS}

The formalism developed in Secs. II and IV has been applied to calculate positron emission in 
various heavy-ion-collision processes. The required amplitudes entering (2.22)-(2.24) were calculated both in time-dependent perturbation theory up to second order and by numerical solution of the coupled differential equations (2.7). In the following we will first discuss the approximations inherent in our model and then briefly discuss the perturbative results. The main emphasis will be placed on the subsequent presentation of the coupled-channel calculations for positron creation in both subcritical and supercritical collisions.

\section{A. Approximations}

In order to arrive at numerical results, several approximations have been applied to the semiclassical quasimolecular model under discussion. They are as follows.

(1) While the (nonseparable) two-center Dirac equation has been solved by Müller et al. for bound states, ${ }^{58-60}$ no solutions are available at present for the relativistic molecular continuum. Detailed comparisons of binding energies and coupling matrix elements have shown, however, that up to internuclear distances $>500 \mathrm{fm}$ the inner-shell states are well described by restriction to the $l=0$ part in a multipole expansion of the two-center potential. ${ }^{61}$ Even for not too asymmetric heavy-ion systems, $\left|Z_{1}-Z_{2}\right| /\left(Z_{1}+Z_{2}\right)$ $<0.2$ the monopole term was found to be dominant. For the spherically symmetric problem, both bound and continuum states are easily generated. Therefore all calculations presented in this paper will be done by use of the monopole approximation. Owing to the spherical symmetry of $V_{0}(r, R)$, rotational coupling acts only within angular momentum multiplets and does not give rise to excitations. Also electron promotion cannot be described by the monopole approximation.

(2) The calculations are restricted to $\kappa=-1$ and +1 states $\left(n s_{1 / 2}\right.$ and $\left.n p_{1 / 2}\right)$. Both sets are decoupled since they have different parity. They are expected to be the dominant channels on theoretical grounds, since in the superheavy systems under consideration the wave functions with $|\kappa|=1$ are most severely distorted by the strong potential, leading to large coupling matrix elements.

(3) It is well known ${ }^{62}$ that the quasimolecular (PSS) model suffers from spurious asymptotic $\partial / \partial R$ couplings: Since the basis states are calculated under the assumption of fixed nuclei, they do not satisfy the correct boundary conditions. With respect to this basis, the nonvanishing nuclear velocity $\dot{R}$ induces transitions at arbitrarily large distance. This problem has been discussed extensively in the literature. ${ }^{63-67}$ It may be avoided by introducing electron translation factors, which asymptotically switch over the basis to "traveling orbitals" correlated to either of the moving nuclei. Various problems are associated with this procedure, especially when continuum states are involved. For a detailed discussion compare the work of Heinz. ${ }^{68}$

In the present calculations we have simulated translation effects in a crude manner: All coupling matrix elements are damped off at separations $R \sim 1500, \ldots, 2000 \mathrm{fm}$ using a Gaussian factor. Compared to the nonrelativistic case (e.g., $p-H$ collisions) relativistic quasimolecular systems exhibit a strong maximum of the radial coupling matrix elements at small $R$ where most of the excitation takes place. Therefore translational effects should be somewhat less critical here. The results of Ref. 68 indicate, however, that future calculations have to carried out to larger distances and employ more realistic asymptotic corrections.

(4) We neglect all effects due to the electronelectron interaction. Rihan et al. ${ }^{69}$ have argued that the relaxation times for the nondiagonal part of this interaction is larger than the collision time. A reliable assessment of the diagonal part, i.e., screening effects, is difficult since the electron shells are dynamically excited in the course of the collision and the outer electrons will not be adiabatic. Fully relaxed molecular HartreeFock calculations, which have been performed recently for superheavy systems, ${ }^{70}$ therefore may overestimate the effect.

Investigations of inner-shell and positron excitation in the framework of the presented model using a simple Thomas-Fermi screening function lead to somewhat enhanced probabilities without change of the general characteristics. ${ }^{71}$ A substantial reduction of the critical distances ${ }^{58,59,72,73}$ due to electron screening has been found in Ref. 74 from relativistic Hartree-Fock-Slater calculations in the monopole approximation. The results presented below therefore are to be considered to give an "upper bound" for the influence of level diving in supercritical collisions.

\section{B. Time-dependent perturbation theory}

Assuming weak coupling the solutions of the system of differential equations (2.7) may be reduced to simple time integrals. Taking $a_{i i} \sim 1$ and $a_{i j}$ $\ll 1$ for $i \neq j$ the amplitude at the rhs of (2.7) is approximated by a constant and we obtain the first-order result

$a_{i j}^{(1)}(t)=-\int_{-\infty}^{t} d t^{\prime}\left\langle\phi_{j}\left|\frac{\partial}{\partial t^{\prime}}+i H\right| \phi_{i}\right\rangle e^{-i\left[x_{i}\left(t^{\prime}\right)-x_{j}\left(t^{\prime}\right)\right]}$. 
Thus the transition amplitude is a quasi-Fourier transform (generally with variable frequency) of the coupling matrix element between the initial and final states. Since the integrand depends parametrically on time via $R(t)$, the result is sensitive to the nuclear trajectory.

Pair production according to (5.1) in the quasimolecular monopole model was first calculated in Ref. 75. Owing to the deformation of the continuum states at small internuclear distance, rather high production rates and, above all, a very strong dependence on nuclear charge $\sigma^{e^{+}}$ $\propto\left(Z_{1}+Z_{2}\right)^{18}$ was predicted.

The general structure of the Fourier integral (5.1) can be used for a qualitative understanding of the excitation process as was first pointed out by Bang and Hansteen. ${ }^{24}$ For instance, the typical collision frequency will be of the order $\omega=v / R_{0}$, where $R_{0}$ is the distance of closest approach and $v$ the bombarding velocity. The integral (5.1) cannot be done analytically even in the monopole model but an approximate solution was given by Refs. 76. Similar, but slightly modified solutions were later presented by 77, and 78 (see also Ref. 79). It turns out that the transition probability depends, in good approximation, exponentially on the ratio $\Delta E_{i j}^{0} / \hbar \omega=\Delta E_{i j}^{0} R_{0} / \hbar v$, where $\Delta E_{i j}^{0}$ is the transition energy at distance $R_{0}$. Upon integration over the energy of the ejected electron, scaling laws can be deduced for the excitation probability as a function of impact parameter and, after a further integration, for the excitation cross section.

The scaling behavior is well reflected in the experimental data on $K$-hole formation ${ }^{80-86}$ and positron creation..$^{87-91}$ It gives insight into the kinematic aspects of the excitation mechanism. One has to keep in mind, however, the failure of perturbation theory to account for the observed large excitation rates. This is attributed to multistep processes as discussed below.

Before we turn to the full coupled-channel calculations we briefly discuss the extension of perturbation theory. Equation (5.1) describes transitions between the positron continuum and states above the Fermi level, i.e., higher bound states and continuum electrons. Inner-shell states do not contribute in first order. Since the investigation of the role they play in the positron production process is a main goal of this work we have pushed the analysis to higher (at least second) order. By successive approximation the two-step amplitude (intermediate state $k$ ) reads

$$
\begin{aligned}
a_{i j ; k}^{(2)}(t)=-\int_{-\infty}^{t} d t^{\prime} & \left\langle\phi_{j}\left|\frac{\partial}{\partial t^{\prime}}+i H\right| \phi_{k}\right\rangle \\
& \times e^{-i\left[x_{k}\left(t^{\prime}\right)-x_{j}\left(t^{\prime}\right)\right]} a_{i k}^{(1)}(t) .
\end{aligned}
$$

The total transition amplitude is given by the coherent sum over all contributions

$$
a_{i j} \simeq a_{i j}^{(1)}+\sum_{k} a_{i j ; k}^{(2)} .
$$

In this approach we have calculated pair creation in subcritical systems in the angular momentum channels $\kappa=-1\left(s_{1 / 2}\right)$ and $\kappa=+1\left(p_{1 / 2}\right)$. Direct and two-step transitions via the three innermost bound states for each $\kappa$ have been added. An inclusion of continuum intermediate states would mainly result in a shift of the electron spectrum. ${ }^{41}$ The results were given in Refs. 92 and 26 and only the main features shall be summarized here..$^{93}$

(i) $s(\kappa=-1)$ and $p_{1 / 2}(\kappa=+1)$ waves contribute roughly equally to positron production.

(ii) While the amplitudes $a_{i j}^{(1)}(\infty)$ are purely imaginary (provided the phases $\chi_{k}$ are chosen symmetric with respect to $t=0$, the turning point of the trajectory), the second-order amplitudes $a_{i j ; k}^{(2)}(\infty)$ will have a real part. The relative phase angle was found to increase with the binding energy of the intermediate state $k .^{92}$ This is illustrated in Fig. 8 for the six innermost $s$ and $p_{1 / 2}$ states in a central $5.9 \mathrm{MeV} / \mathrm{u} \mathrm{Pb}-\mathrm{Pb}$ collision. The displayed amplitudes have to be added coherently, $\kappa=-1$ and +1 separately .

(iii) Although being of higher order, the contributions of the $1 s$ and $2 p_{1 / 2}$ states are comparable in magnitude to the direct pair-creation process. They grow particularly fast with nuclear charge and constitute the largest single components for systems heavier than $Z_{1}+Z_{2} \sim 175$.

(iv) The kinematic characteristics of direct and two-step excitations do not differ much. In both cases the same amount of energy has to be transferred in a similar region of space and time.

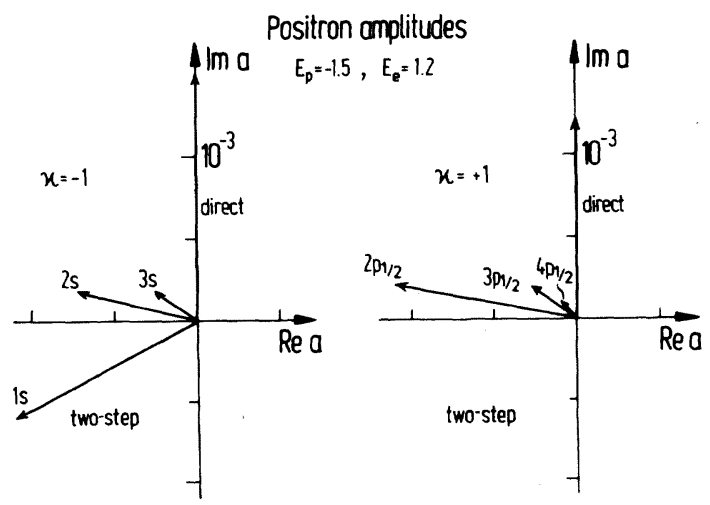

FIG. 8. The complex pair formation amplitudes in a central $5.9 \mathrm{MeV} / \mathrm{u} \mathrm{Pb}-\mathrm{Pb}$ collision, calculated in firstand second-order perturbation theory including several intermediate states. Left half: $s_{1 / 2}$ waves, right half: $p_{1 / 2}$ waves. 


\section{Coupled-channel calculations}

The perturbative calculations briefly reported in Sec. VB describe much of the physics involved in the excitation process. The strong dependence on impact parameter and collision energy could not be understood without use of the quasimolecular picture. The large magnitude of the excitation rates and their $Z$ dependence are characteristic for relativistic effects in the wave function, in particular the loss of any atomic length scale other than the nuclear separation distance.

The above-mentioned growing importance of the second-order term in (2) for heavy systems already indicates, however, that perturbation theory is of limited validity. Furthermore the remaining discrepancy between predicted ${ }^{94,95}$ and observed ${ }^{80-86} K$-vacancy probability may be explained in this way. Owing to the large values of the radial coupling matrix elements at small internuclear distance multistep excitations cannot be neglected. As shown in Ref. 41 a coherent superposition of the various contributions gives an increase of $P_{1 s \sigma}$ by a factor of typically 3 to 5 over first-order direct-ionization calculations, in general agreement with experiment. All multistep processes are properly incorporated if the amplitudes $a_{i j}$ are obtained by direct solution of the coupled differential equations (2.7).

We have solved the system of differential equations including up to eight bound states and $\sim 15$ states in the upper continuum, separately for $\kappa=+1$ and -1 . The integration was performed with a standard Hamming predictor-corrector routine taking about 1500 steps in time. It proved advantageous to employ the symmetry relation (2.14) (valid for time-symmetric nuclear trajectories) and compute

$$
\bar{N}_{E_{p}}=\sum_{i>F}\left|a_{i, E_{p}}\right|^{2}=\sum_{i>F}\left|a_{E_{p}, i}\right|^{2}
$$

Since the probability for positron excitation is small compared to unity, it is sufficient to include only one state at a time when varying $E_{p}$, i.e., the lower continuum can be coupled in perturbation theory.

Now we will analyze coupled-channel calculations in four different heavy-ion-collision systems, $\mathrm{Pb}-\mathrm{Pb}, \mathrm{Pb}-\mathrm{U}, \mathrm{U}-\mathrm{U}, \mathrm{U}-\mathrm{Cf}$ with the total charges $Z_{1}+Z_{2}=164,174,184$, and 190. For reference, Fig. 9 shows the binding energies used in the calculations for the two lowest states. Table III gives the predicted total probability for positron production in head-on collisions $(b=0)$. The impact energy per nucleon was kept constant at $E_{1 \mathrm{ab}}=5.9 \mathrm{MeV} / \mathrm{u}$, corresponding to a nuclear velocity of about $v / c \sim 0.113$. The table lists the re-

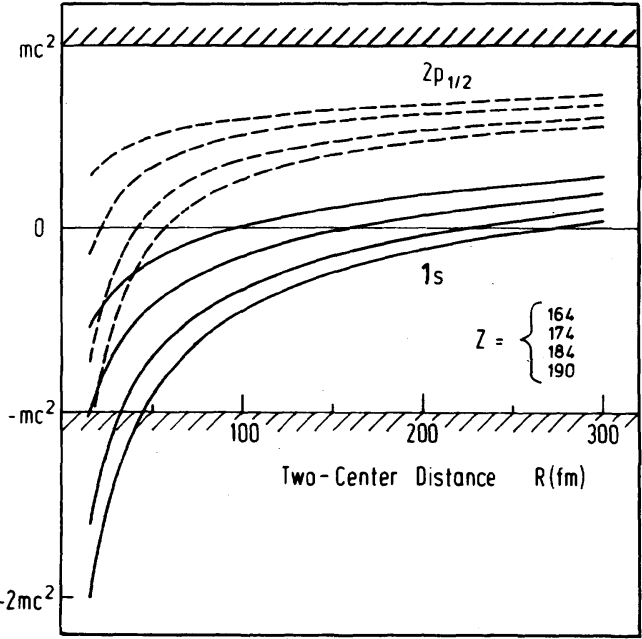

FIG. 9. Energies of the $1 s \sigma$ and $2 p_{1 / 2 \sigma}$ states as a function of internuclear distance $R$ in the systems $\mathrm{Pb}-\mathrm{Pb}$, $\mathrm{Pb}-\mathrm{U}, \mathrm{U}-\mathrm{U}$, and $\mathrm{U}-\mathrm{Cf}$, calculated in monopole approximation for extended nuclei. The latter two systems are supercritical.

sults for various positions of the Fermi level: $F=0$ corresponds to fully stripped nuclei, for $F=3$ the three lowest $n s \sigma$ and $n p_{1 / 2 \sigma}$ states are occupied before the collision, and for $F=N$ only the upper continuum is available for excitation of the electron. (Of course, bound states still can act as intermediate states in the excitation process.) For comparison also the direct plus two-step perturbative results described in the last paragraph are shown for the two lighter systems. In the same manner Table IV gives the total cross sections $\sigma^{e^{+}}$in units of $\mathrm{mb}$.

An analysis of the data contained in these tables leads to the following observations: (1) The results obtained by the coupled-channel method are larger compared with perturbation theory. The enhancement factor is smaller, however, than that found for inner-shell hole production. (2) The production rates increase very fast with total nuclear charge, flattening somewhat for the highest- $Z$ values. If parametrized by a power law $\left(Z_{1}+Z_{2}\right)^{n}$ the power takes values of 20 down to 13 $(F=3)$. If the distance of closest approach $R_{0}$ is kept fixed instead of the impact velocity, $n$ becomes still larger. (3) In collisions of bare nuclei $(F=0)$ positron production is increased by up to two orders of magnitude. Here also the $Z$ dependence is extremely steep $(n \simeq 29)$. Mainly responsible for this effect is the contribution of the $1 s$ state which in normal collisions $(F>0)$ is suppressed due to the small $K$-vacancy probability. If the $K$ shell is empty it becomes the dominant final state for pair production. This clearly reflects the strong coupling between the $1 s$ state and 
TABLE III. Probability $P^{e^{+}}(0)$ for positron emission calculated by solution of the coupledchannel equations for head-on collisions of $\mathrm{Pb}-\mathrm{Pb}, \mathrm{Pb}-\mathrm{U}, \mathrm{U}-\mathrm{U}$, and $\mathrm{U}-\mathrm{Cf}$ with bombarding energy $5.9 \mathrm{MeV} / \mathrm{u}$. Compared are the results assuming different positions of the Fermi level $F$ and values from first-plus second-order perturbation theory.

\begin{tabular}{cllll}
\hline \hline & \multicolumn{4}{c}{$Z_{1}+Z_{2}=$} \\
& 164 & 174 & 184 & 190 \\
\hline 0 & $4.26(-4)$ & $2.37(-3)$ & $1.18(-2)$ & $2.83(-2)$ \\
3 & $4.03(-5)$ & $1.30(-4)$ & $3.69(-4)$ & $5.62(-4)$ \\
$N$ & $3.35(-5)$ & $1.05(-4)$ & $2.68(-4)$ & $4.08(-4)$ \\
$\begin{array}{c}\text { Perturbation } \\
\text { theory }\end{array}$ & $2.8(-5)$ & $8.5(-5)$ & & \\
\hline \hline
\end{tabular}

the antiparticle continuum which it approaches and even enters in the supercritical region.

In all cases investigated the channels $\kappa=-1$ and +1 contribute about equally to the total result. In constrast to situations where perturbation theory is valid, an analysis of the coupled-channel results to determine individual contributions is less satisfactory. Since all bound and positive energy continuum states mutually are strongly coupled, it is not well justified to separate single levels or interactions. Nevertheless, we have made some restricted calculations, leaving out parts of the coupling. To be specific, we give some results for $5.9 \mathrm{MeV} / \mathrm{u}$ central collisions of $\mathrm{Pb}-\mathrm{Pb}$. The positron production probability (in the $s_{1 / 2}$ channel only) is $P^{e^{+}}=2.1 \times 10^{-5}$ for the Fermi surface $F=3$. In comparison to this result of the fully coupled calculation we obtain (i) $31 \%$ if only direct transitions to the upper continuum are included, (ii) $17 \%$ from the $1 \mathrm{~s}$ state alone, no higher bound states included [this corresponds to the two-step process of Eq. (5.2)], (iii) $49 \%$ from the $1 \mathrm{~s}$ state alone, which, however, is fully coupled to the higher states; the increase of $1 s \sigma$ vacancy production shows up in the positron rate, and (iv) $68 \%$ from a fully coupled calculation excluding the $1 s$ state.

Further results of the model are contained in the following figures: Figure 10 shows the energy spectra of positrons $d P / d E_{p}$ produced in 5.9

TABLE IV. Same as Table III for the total positron cross section $\sigma e^{+}$in units of $\mathrm{mb}$.

\begin{tabular}{ccccr}
\hline \hline \multicolumn{5}{c}{$Z_{1}+Z_{2}=$} \\
\hline & 164 & 174 & 184 & 190 \\
0 & 4.5 & 24.0 & 120 & 300 \\
3 & 0.27 & 0.87 & 2.2 & 3.4 \\
$N$ & 0.22 & 0.63 & 1.55 & 2.4 \\
$\begin{array}{c}\text { Perturbation } \\
\text { theory }\end{array}$ & 0.20 & 0.52 & & \\
\hline \hline
\end{tabular}

$\mathrm{MeV} / \mathrm{u}$ head-on collisions $(F=3)$. As is well known, the emission of low-energy positrons is suppressed by Coulomb repulsion while at high energies the spectra fall off exponentially in analogy to the spectra of $\delta$ electrons. The shapes do not differ qualitatively from the perturbative results. Obviously they are practically independent of the charge of the collision system except for a minute shift of the maximum which lies at about $450 \mathrm{keV}$ kinetic energy. Figures 11(a) and 11(b) gives the impact-parameter dependence of positron production. The abscissa is the distance of closest approach $R_{\min }$ which is related to impact

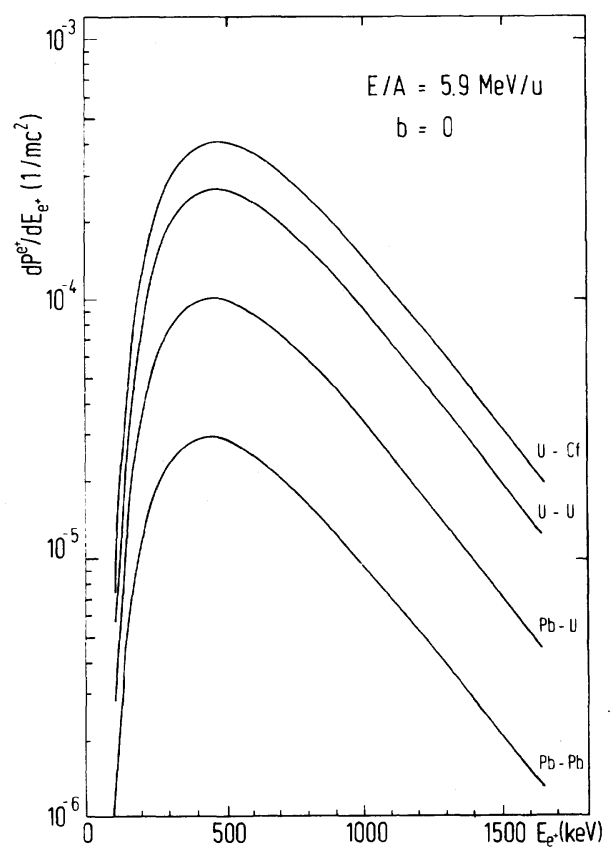

FIG. 10. Energy spectra of positrons created in 5.9 $\mathrm{MeV} / \mathrm{u}$ head-on collisions of $\mathrm{Pb}-\mathrm{Pb}, \mathrm{Pb}-\mathrm{U}, \mathrm{U}-\mathrm{U}$, and $\mathrm{U}-\mathrm{Cf}$. The results are calculated in the coupled-channel approach assuming initial occupation up to the states $3 s \sigma$ and $4 p_{1 / 2 \sigma}(F=3)$. 

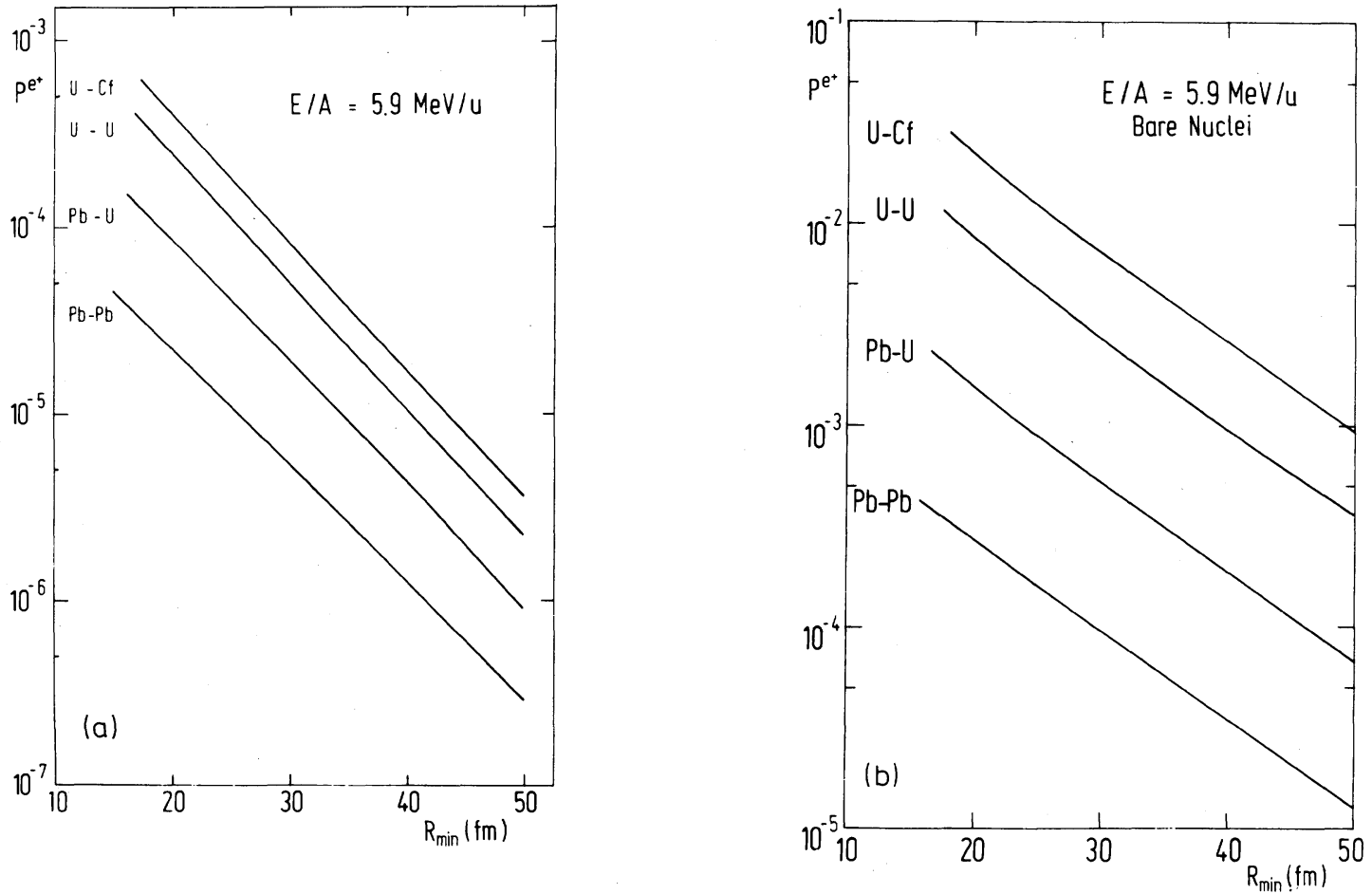

FIG. 11. (a) Emission probability of positrons in $5.9 \mathrm{MeV} / \mathrm{u}$ collisions as a function of distance of closest approach $R_{\min }(F=3)$. (b) Same as (a) for collisions of totally stripped heavy ions. The large enhancement is mainly due to the contribution of the $1 s \sigma$ state.

parameter $b$ by $R_{\mathrm{min}}=a\left[1+\left(1+(b / a)^{2}\right)^{1 / 2}\right]$. A nearly perfect exponential decrease of the curves is noted. The high value of the slope is understandable in terms of the energy transfer required to produce a pair. Therefore the impact-parameter dependence is much weaker in the case $F=0$ [Fig. 11(b)] where the gap between the lowest empty state $(1 s \sigma)$ and the positron continuum becomes small or vanishes. For the collisions with initially occupied inner shells the slope of $P^{e^{+}}\left(R_{\min }\right)$ becomes steeper for the heavier systems.

\section{Discussion of the results}

The results displayed in the last two subsections demonstrate remarkable features of the pairproduction mechanism in "slow" collisions of highly charged nuclei. In particular, the large excitation rates and their high sensitivity on total nuclear charge in the region $Z_{1}+Z_{2} \simeq Z_{\mathrm{cr}}$, are characteristic for the action of the time-dependent strong Coulomb field.

One question must be studied in more detail: What is the influence of the "diving" of the $1 \mathrm{~s}$ level in supercritical collisions on positron production? The energy spectra and impact-parameter dependence, depicted in Figs. 10 and 11, have already shown that our theory does not predict any drastic change of observables at the border of the supercritical region, but rather a smooth increase of production rates with $Z$. This seems to be at variance with the results of Sec. IV C, where an additional coupling between $1 s$ state and positron continuum emerged.

To study its influence more closely we have performed calculations where the matrix element $\left\langle\phi_{E_{p}}|H| \Phi_{R}\right\rangle$ was artificially switched off. Figure 12 compares the resulting positron emission probabilities in U-U and U-Cf collisions calculated with and without the spontaneous coupling. At large scattering angles (small impact parameters) the values of $P^{e^{+}}$are significantly reduced when this coupling is omitted. This becomes even more obvious in the positron spectra which are shown for head-on collisions in Fig. 13. The dashed curves are much depressed in the region of positron energies where, in the supercritical phase of the collision, the resonance is located. In the heavier system the shape of the spectrum is also drastically altered.

In the first place, this result gives confidence in the employed projection method; the superposition of two couplings which by themselves lead to totally different results produces spectra and excitation rates, which are a smooth continuation of the corresponding quantities in the subcritical 


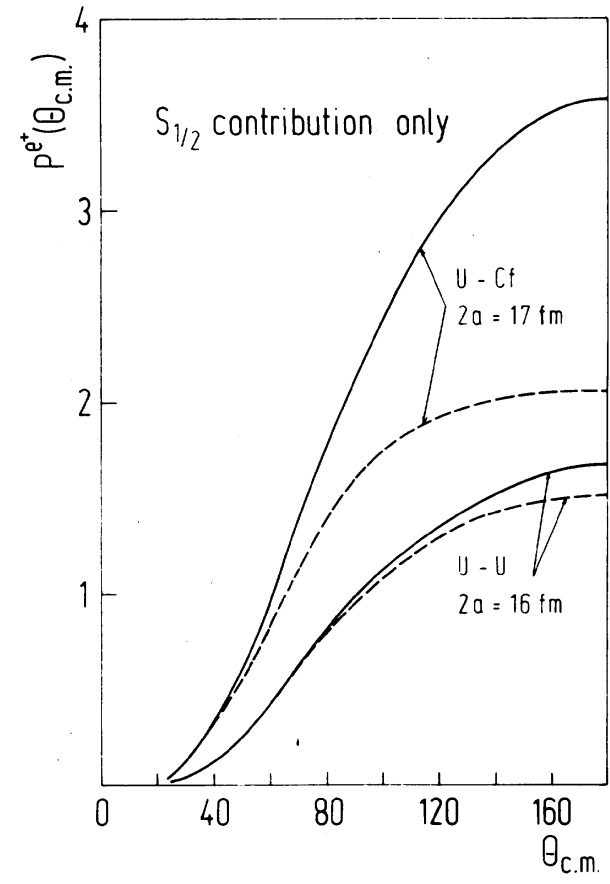

FIG. 12. Probability of positron emission in the $s_{1 / 2}$ channel as a function of c.m. scattering angle in collisions of U-U at $2 a=16 \mathrm{fm}$ and U-Cf at $2 a=17 \mathrm{fm}$. Full lines: Fully coupled calculation. Dashed lines: The spontaneous coupling $\left\langle\tilde{\phi}_{E_{p}}|H| \Phi_{R}\right\rangle$ has been omitted.

region (cf. Figs. 10 and 11). An explanation for this result can be gained by looking at the structure of the coupling matrix elements as a function of time. In Fig. 14 the values of $\left\langle\tilde{\phi}_{E_{p}}|\dot{R} \partial / \partial R| \Phi_{R}\right\rangle$ and $\left\langle\tilde{\phi}_{E_{p}}|H| \Phi_{R}\right\rangle, E_{p}=-2$, are drawn for a head-on $\mathrm{U}-\mathrm{Cf}$ collision with $2 a=18 \mathrm{fm}$. For comparison the corresponding radial coupling in $\mathrm{Pb}-\mathrm{Pb}$ collisions is included in the graph. Obviously, the spontaneous coupling is compensated by a corresponding reduction of the $1 s$-induced contribution. Both couplings have to be added coherently
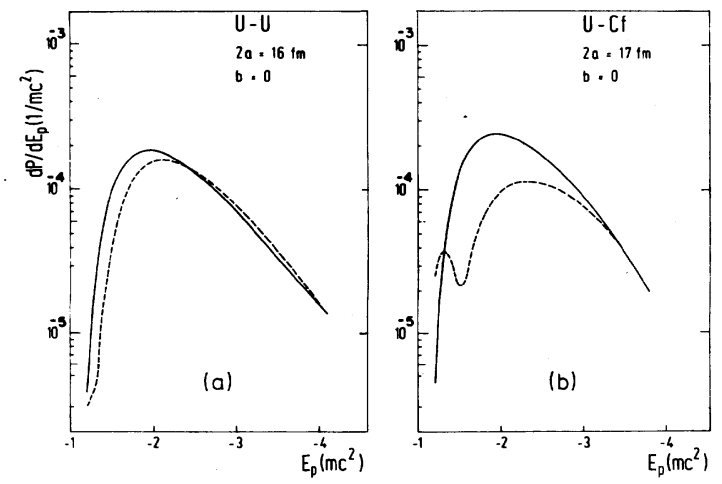

FIG. 13. The positron spectra in U-U and U-Cf collisions. Meaning of the curves as in Fig. 12.

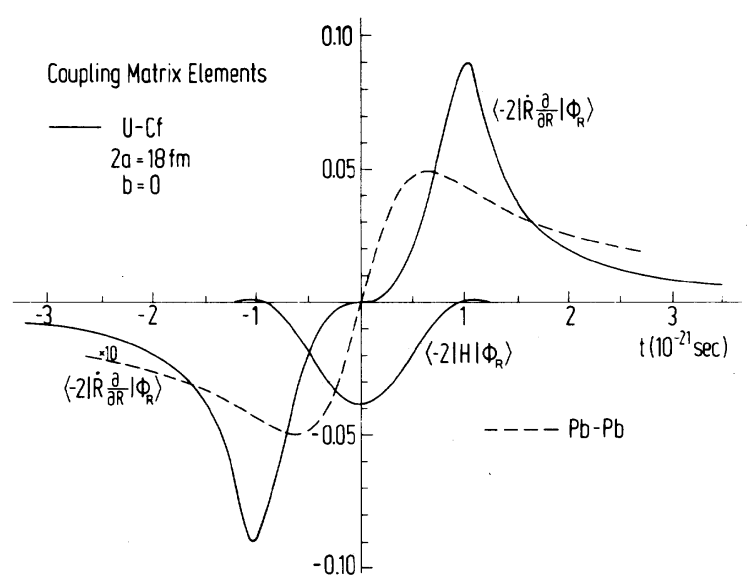

FIG. 14. Matrix elements of the induced and spontaneous positron coupling as a function of collision time in central collisions of U-Cf, $2 a=18 \mathrm{fm}$. For comparison, the dashed line shows the radial coupling in a $\mathrm{Pb}-\mathrm{Pb}$ collision (multiplied by a factor of 10 ).

[with a relative phase factor of $i$, cf. (4.25)], leading to excitation rates which do not differ qualitatively from the subcritical results.

This observation is in general agreement with the notion of a "dynamical width" which may be associated with a collision time $\tau$ by the uncertainty principle $\Gamma_{\mathrm{dyn}} \simeq \hbar / \tau$ and which leads to a smooth transition between subcritical and supercritical collisions. The shortness of $\tau$ prevents any sudden threshold effects and in particular precludes the existence of sharp structures in the positron spectra near the diving energy $E_{1 s}\left(R_{\text {min }}\right)$. A proof of supercriticality of a given collision, therefore, according to our calculations will not be possible by the observation of qualitative features in the excitation rates. Rather, a quantitative analysis is needed, including the rate of $1 s \sigma-$ vacancy formation, which is sensitive to the binding energy.

A unique signature for spontaneous positron production could be gained in collisions with prolonged interaction time. Rafelski, Müller, and Greiner suggested the use of deep inelastic nuclear collisions ${ }^{96}$ to keep the nuclei in close contact for some delay time $T$. While the radial coupling is small during this period, $\dot{R} \ll v_{\infty}$, the decay coupling $\left\langle\tilde{\phi}_{E_{P}}|H| \Phi_{R}\right\rangle$ remains constant at its maximum value. This leads to an increase of positroncreation rates as a function of $T$. In the (hypothetical) limit of total fusion to a long-lived supercritical compound nucleus, a positron line with the natural decay width (4.26) would emerge.

Coupled-channel calculations within the framework of the theory developed in this paper ${ }^{97}$ have lead to the conclusion, that time delays in the region of $2, \ldots, 3 \times 10^{-21} \mathrm{~s}$ are required to get a 
clear distinction of the diving process. ${ }^{98}$ If the background due to nuclear excitation is separable, and if collisions with sufficiently long reaction times can be selected, this experiment could give an unequivocal answer to the question of the decay of the neutral vacuum.

\section{E. Comparison with experiment}

Since beams of very heavy ions at energies close to the Coulomb barrier have become available at GSI (Darmstadt), a number of experiments have been performed to study positron production in highly charged collision systems. The search for such processes has been largely successful. A major problem in analyzing the experiments consists in the background originating from nuclear processes. Already well below the barrier the nuclei can be excited by Coulomb excitation. Photons with energy larger than $1022 \mathrm{keV}$ can undergo pair conversion. Although this process takes place long after the collision $\left(\simeq 10^{-13} \mathrm{~s}\right)$, it cannot be distinguished experimentally from the quasimolecular mechanism by ordinary methods. ${ }^{100}$ For nuclei with a simple level structure (e.g., ${ }^{208} \mathrm{~Pb}$ ) the Coulomb excitation can be calculated. The resulting pair creation can be deduced from the theoretically known ${ }^{101}$ conversion coefficients. ${ }^{102}$

Otherwise one has to measure the $\gamma$-spectrum ${ }^{103}$ and fold it with the conversion coefficient. Here the $\gamma$-ray multipolarity has to be known or assumed. Monopole conversion cannot be handled by this method. The procedure was tested in collisons with lighter targets where it quantitatively accounts for the total observed positron production. No significant contribution of atomic positrons is expected in these collisions. Beginning in the region $Z_{1}+Z_{2} \geq 160$ all experiments have found an increase which could not be explained by nuclear conversion.

We will now compare the experimental data published so far with the predictions of theory. We adopt the coupled-channel results, assuming $F=3$, i.e., the states above $3 s \sigma$ and $4 p_{1 / 2 \sigma}$ are empty (this choice should give an upper bound for the production rates). Figure 15 shows the result of Kozhuharov et al. ${ }^{88}$ for three collision systems $\mathrm{Pb}-\mathrm{Pb}, \mathrm{U}-\mathrm{Pb}$, and $\mathrm{U}-\mathrm{U}$, at $5.8 \mathrm{MeV} / \mathrm{u}$, measured with an orange-type $\beta$ spectrometer. The probability of positron emission in a narrow energy window around $490 \mathrm{keV}$ is shown as a function of projectile center-of-mass (c.m.) scattering angle. Here and in the following figures projectile and target nuclei are not distinguished. The theoretical curves therefore have been symmetrized with respect to forward and recoil scattering. The shape of the theoretical curves is in good agree-

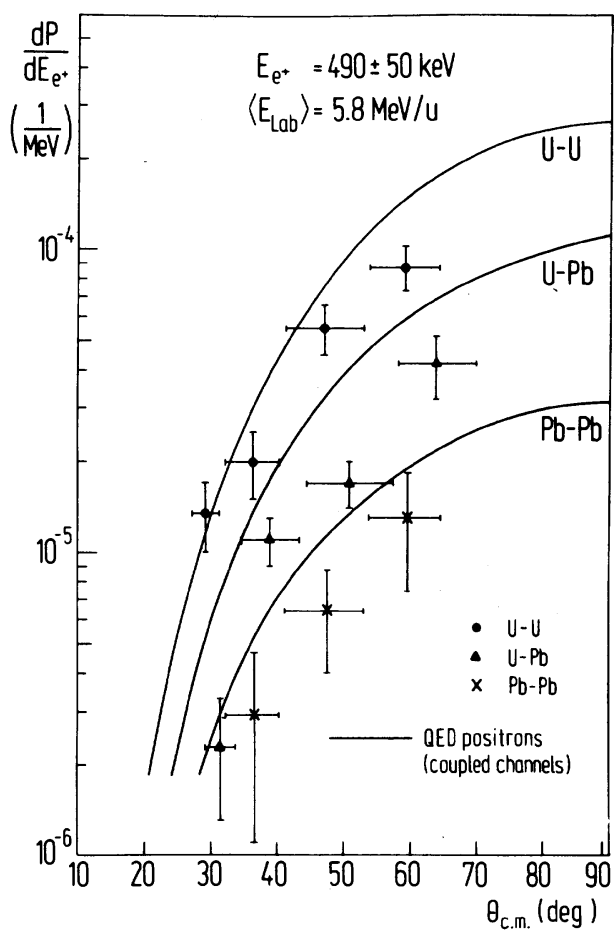

FIG. 15. Positron-production probability in an energy window $E_{e}=490 \pm 50 \mathrm{keV}$ as a function of projectile scattering angle in $5.8 \mathrm{MeV} / \mathrm{u} \mathrm{Pb}-\mathrm{Pb}, \mathrm{U}-\mathrm{Pb}$, and $\mathrm{U}-\mathrm{U}$ collisions. Experimental data taken from Kozhuharov et al. (Ref. 88). The nuclear background is subtracted.

ment with experimental data. Also the predicted increase of positron production with charge $Z=$ $Z_{1}+Z_{2}$ by nearly an order of magnitude (while $\Delta Z / Z$ is only $12 \%$ ) is fully confirmed by the measurement. The absolute magnitude of the theoretical values, however, is generally too high.

In another, independent experiment using a solenoidal spectrometer Backe et al.$^{87,104}$ obtained differential and integrated positron probabilities for various impact energies. Figure 16 shows $P^{e^{+}}\left(R_{\mathrm{min}}\right)$ for the three systems already discussed and in addition for the heaviest accessible system $\mathrm{U}-\mathrm{Cm}(Z=188)$. The scattered particle was detected in a fixed angular window $\theta_{\text {lab }}=45^{\circ} \pm 10^{\circ}$ so that the various values of $R_{\min }$ were obtained by variation of the collision energy. The theoretical values are symmetrized and averaged over the region of impact parameters defined by the experimental angular window.

Again, a general agreement is found in the $Z$ and $\boldsymbol{R}_{\min }$ dependence. In particular the $\mathrm{Pb}-\mathrm{Pb}$ results are explained even quantitatively, in contrast to the experiment discussed above. In the heavier systems theory again has a tendency to overestimate the measured data. In addition the experimental slopes are somewhat steeper than predicted. 


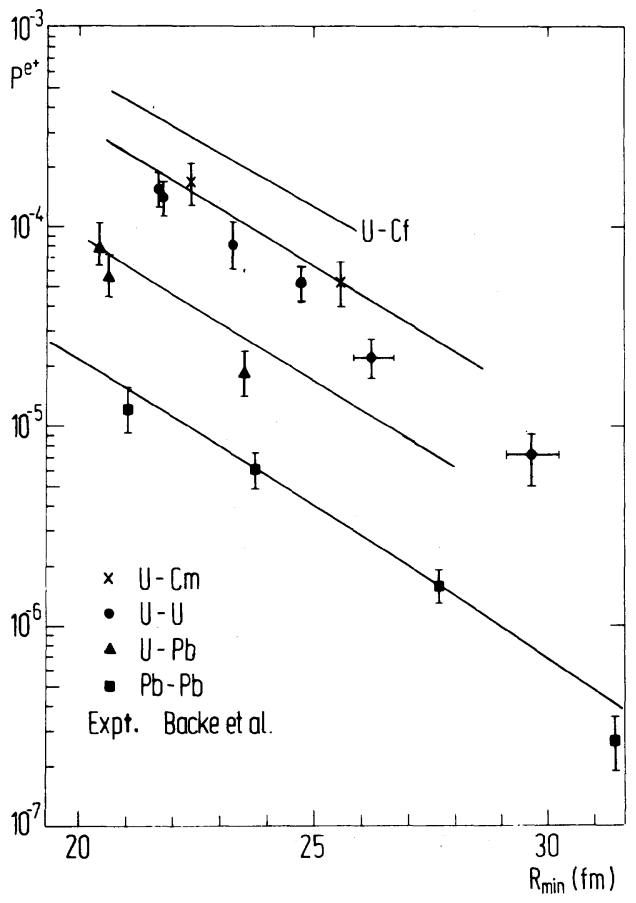

FIG. 16. Positron emission probability for various heavy-ion collision systems, drawn as a function of the distance of closest approach. The scattered ion is detected in an angular window $\theta_{1 \mathrm{ab}}=45^{\circ} \pm 10^{\circ}$. The bombarding energy is varied. Data are taken from Backe et al. (Refs. 87 and 104). The nuclear background is subtracted.

Such a trend seems to be present also in new experimental data.90,105,106 A new generation of experiments was set up to extract the most sensitive information: the energy spectra of positrons, measured in coincidence with the scattered ions. Their knowledge is most useful if one wants to verify the theoretical predictions or find deviations hinting to the positron-creation mechanism. Figure 17 shows the first published positron spectra of Backe et al.$^{89}$ for $5.9 \mathrm{MeV} / \mathrm{u} \mathrm{U}-\mathrm{Pd}, \mathrm{U}-\mathrm{Pb}$, and $\mathrm{U}-\mathrm{U}$ collisions. The U-Pd $(Z=138)$ positrons can be fully accounted for by nuclear conversion (thin curves). In the system $\mathrm{U}-\mathrm{Pb}$ the sum of background and calculated QED positron rates (full curve) is in excellent agreement with the observed spectrum. The spectrum of the U-U system is explained less closely. Its maximum seems to be shifted to lower kinetic energies. Again, such a tendency seems to be observed in several experiments with $\mathrm{U}-\mathrm{U}$ and $\mathrm{U}-\mathrm{Cm}$ currently under way at GSI. ${ }^{104-106}$

\section{SUMMARY}

We have studied the mechanism of pair production in collisions of very heavy ions within the framework of a dynamical theory of excitation

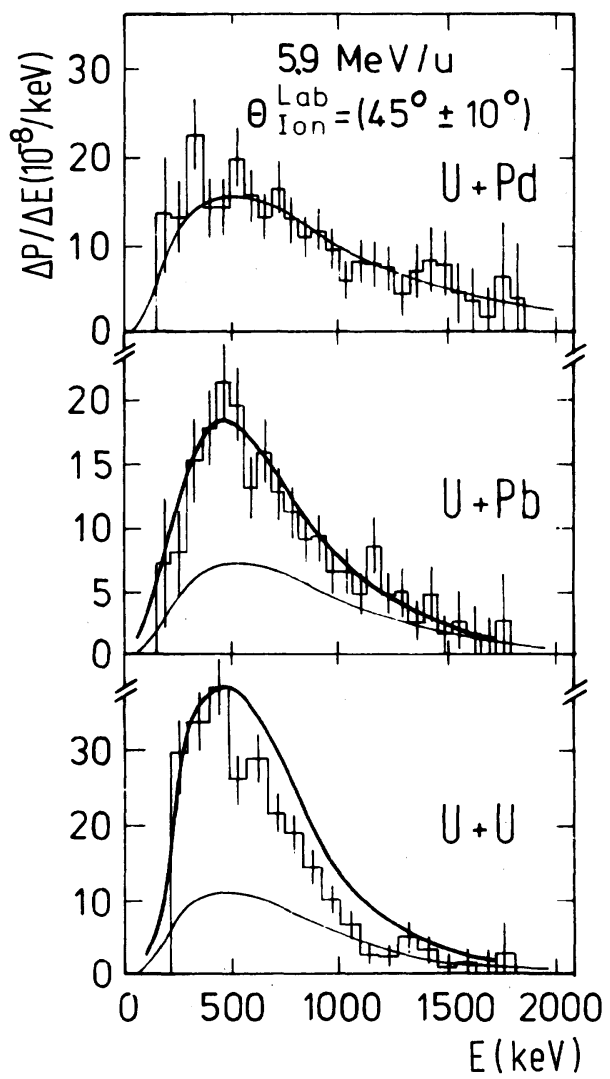

FIG. 17. Spectra of emitted positrons in $5.9 \mathrm{MeV} / \mathrm{u}$ collisions measured by Backe et al. (Ref. 89) in coincidence with ions scattered in the angular window $\theta_{1 \mathrm{ab}}$ $=45^{\circ} \pm 10^{\circ}$. The spectrum in the lightest system, U-Pd, is explained by nuclear pair conversion alone (thin line). In the $\mathrm{U}-\mathrm{Pb}$ and $\mathrm{U}-\mathrm{U}$ systems the sum of nuclear and calculated atomic positron probabilities (heavy lines) is shown.

based on the quasimolecular picture. Massive relativistic effects in the wave functions of electrons and positrons in $s_{1 / 2}$ and $p_{1 / 2}$ states, caused by the coherent action of the Coulomb field generated by the two nuclei, are reflected in the excitation rates. As known already from the process of inner-shell vacancy creation, positron formation is concentrated in a region of close collisions, i.e., high impact energies and small impact parameters.

The most outstanding result is a very steep increase of positron production with nuclear charge, which alone makes the experimental observation possible against a large background. Contrary to the case of light collision systems, theory predicts the sharply growing importance of the inner-shell bound states ( $1 s$ and $2 p_{1 / 2}$ ) in the pair-production mechanism if the supercritical region is approached. If prepared empty, the $1 s$ state will be the dominant final state for the electron in pair creation since this level interacts strongly with the antiparticle continuum in the course of the collis- 
ion.

We have developed a theory which properly takes into account the resonance character of the dived $1 s$ state. The results of our coupled-channel calculations indicate, that no sharp threshold effects are to be expected at the border of the supercritical region, in accordance with the notion of dynamical collision broadening.

The experiments performed so far have convincingly established the predicted strong increase of positron production in close collisions of heavy ion systems with very high total nuclear charge of heavy-ion systems. There remain some discrepancies with theory in absolute magnitude, in the slope of $P^{e^{+}}(\mathrm{b})$, and, possibly, in the shape of the positron spectra. At present it cannot be determined whether these differences have experimental origins, are caused by the approximations employed in the theoretical model, or do reflect some deviations from the predictions of QED which are of principal interest. Future studies should lift the approximations discussed in Sec. V A and also include effects from field fluctuations like vacuum polarization ${ }^{107}$ and self-energy, ${ }^{108}$ which have been neglected in the present work. An unambiguous demonstration of the decay of the neutral vacuum may be possible, though very difficult, by measuring enhanced positron production in collisions with nuclear contact leading to a sufficiently prolonged interaction time.

\section{ACKNOWLEDGMENTS}

We are grateful to G. Soff and U. Müller for useful conversations. Furthermore we thank $\mathrm{H}$. Backe, H. Bokemeyer, J.S. Greenberg, E. Kankeleit, P. Kienle, C. Kozhuharov, W.E. Meyerhof, D. Schwalm, J. Schweppe, and P. Vincent for many discussions concerning the experiments and for making their experimental data available to us. This work has been supported by the Bundesministerium für Forschung und Technologie (BMFT), the Deutsche Forschungsgemeinschaft (DFG), and the Gesellschaft für Schwerionenforschung (GSI). Also, this work has been partially supported by the Division of Basic Energy Sciences, U.S. Dept. of Energy, under Contract No. W-7405-eng-26 with the Union Carbide Corporation.

\section{APPENDIX: ELECTRON-POSITRON PAIR} CORRELATIONS

To complete the discussion of Sec. II in this appendix we discuss correlations between pairs of emitted electrons and positrons. We will stay within the framework of the monopole approximation. According to the theory presented above, the angular momenta $s_{1 / 2}(\kappa=-1)$ and $p_{1 / 2}(\kappa=+1)$ are the dominant channels. In the experiment, the partial waves will not be distinguished. Therefore the basic equation (2.24) for pair correlations, integrated over the solid angle of the emitted particles, has to be supplemented by terms describing incoherent coincidences. Assuming further, that spin orientations are not measured leads to the result

$$
\begin{aligned}
N_{B_{i}, E_{j}}= & 4\left(N_{E_{i}}^{(-)}+N_{E_{i}}^{(+)}\right)\left(\bar{N}_{E_{j}}^{(-)}+\bar{N}_{E_{j}}^{(+)}\right) \\
& +2\left|\sum_{r<F} a_{r_{i} E_{i}}^{*(-)} a_{r_{j} E_{j}}^{(-)}\right|^{2}+2\left|\sum_{r<F} a_{r, E_{i}}^{*(+)} a_{r, E_{j}}^{(+)}\right|^{2} .
\end{aligned}
$$

Here and in the following the superscripts $(+)$ and (-) will denote the channels $\kappa=+1$ and -1 . The first term in Eq. (A1) is a product of the differential excitation rates for electrons and positrons and thus describes random coincidences. The two remaining terms represent coherent correlations. In particular, they contain the direct transition between the states $E_{j}$ and $E_{i}$. In the limit of lowexcitation rates, Eq. (A1) reduces to (setting $\left.r=E_{j}\right)$

$$
N_{E_{i}, E_{j}} \simeq 2\left|a_{E_{j}, E_{i}}^{(-)}\right|^{2}+2\left|a_{E_{j}, E_{i}}^{(+)}\right|^{2} .
$$

It should be stressed, however, that this simple result is valid only if multiple excitations can be neglected. Since inner-shell bound states are strongly ionized in the collision, the random coincidences will constitute a large part of the total pair correlations. This can make it difficult to extract information from doubly differential measurements.

The information contained in the amplitudes $a_{i j}^{(t)}$ is sufficient to determine also the angular correlation between emitted electrons and positrons. Its measurement has been suggested to obtain additional information on the pair-creation process. ${ }^{77,109}$ To determine the angular correlation, two detectors have to be placed at definite angles so that they can measure the momentum vectors $\overrightarrow{\mathbf{k}}_{e}, \overrightarrow{\mathbf{k}}_{p}$. In such an arrangement partial waves with different angular momentum and parity can interfere. To derive an expression for the number of pairs with electron energy $E_{e}$ and direction $\overrightarrow{\mathrm{k}}_{e} /\left|\overrightarrow{\mathrm{k}}_{e}\right|$ and positron energy $E_{p}$, positron direction $\overrightarrow{\mathbf{k}}_{p} /\left|\overrightarrow{\mathbf{k}}_{p}\right|$, $N_{E_{e}, \overrightarrow{\mathrm{r}}_{e} ; E_{p}, \overrightarrow{\mathrm{k}}_{p}}$, we have to evaluate Eq. (2.24) using number operators for particles (holes) in planewave states instead of the spherical waves used so far. The transformation between the sets of states is a generalization of the Rayleigh plane-wave expansion. It takes the form ${ }^{110,111}$

$$
|\overrightarrow{\mathrm{k}} m\rangle=(4 \pi)^{-1 / 2} \sum_{\kappa \mu}(2 l+1)^{1 / 2} i^{l} e^{-i \delta_{k}}\left(l \frac{1}{2} j \mid O m m\right) D_{\mu m}^{j}|\kappa \mu\rangle,
$$


where $|k m\rangle$ and $|\kappa \mu\rangle$ are plane and spherical waves quantized along the axes $\hat{k}$ and $\hat{z}, D_{\mu m}^{j}$ $\left(\Omega_{\overrightarrow{\mathbf{k}}}\right)$ is the rotation matrix $(\hat{z} \rightarrow \hat{k})$ for angular momentum $j=|\kappa|-\frac{1}{2}$, and $\delta_{k}$ denotes the phase shift due to the potential. The basis states are normalized according to

$$
\begin{aligned}
& \left\langle E^{\prime} \kappa^{\prime} \mu^{\prime} \mid E_{\kappa} \mu\right\rangle=\delta_{\kappa \kappa^{\prime}} \delta_{\mu \prime \mu} \delta\left(E^{\prime}-E\right), \\
& \left\langle\vec{k}^{\prime} m^{\prime} \mid \vec{k} m\right\rangle=\delta_{m^{\prime} m} \delta^{3}\left(\overrightarrow{\mathbf{k}^{\prime}}-\overrightarrow{\mathrm{k}}\right) \text {. }
\end{aligned}
$$

The field operator $\hat{\Psi}$ may be expanded in analogy to $(2.20)$ in the basis of plane-wave states $\phi_{i}^{\prime}$,

$$
\hat{\Psi}=\sum_{i<F} \hat{d}_{i}^{\prime+} \phi_{i}^{\prime}+\sum_{i>F} \hat{b}_{i}^{\prime} \phi_{i}^{\prime}
$$

The canonical transformation between the primed and unprimed particle and hole operators then, is given by (A3), namely,

$$
\begin{aligned}
& \underline{\hat{d}}_{i}^{\prime \dagger}=\sum_{a<F}\left\langle\vec{k}_{i} m_{i} \mid \kappa_{q} \mu_{a}\right\rangle \underline{\hat{d}}_{a}^{\dagger}, \\
& \hat{\underline{b}}_{i}^{\prime}=\sum_{a>F}\left\langle\overrightarrow{\mathrm{k}}_{i} m_{i} \mid \kappa_{a} \mu_{q}\right\rangle \underline{\hat{b}}_{a} .
\end{aligned}
$$

The labels $i$ and $q$ are abbreviations for the sets $\left(\overrightarrow{\mathrm{k}}_{i}, m_{i}\right)$ and $\left(E_{q}, \kappa_{q}, \mu_{\uparrow}\right)$. The number of pairs in the plane-wave states $i$ and $j$ then is given by

$$
\begin{aligned}
& N_{i j}^{\prime}=\left\langle F\left|\underline{\hat{b}}_{i}^{\prime+} \underline{\hat{b}}_{i}^{\prime} \hat{\hat{d}}_{j}^{\prime+} \underline{\hat{d}}_{j}^{\prime}\right| F\right\rangle \\
& =\sum_{a, a^{\prime}, p_{p}, p^{\prime}}\left\langle\kappa_{a} \mu_{a} \mid \overrightarrow{\mathrm{k}}_{i} m_{i}\right\rangle\left\langle\overrightarrow{\mathrm{k}}_{i} m_{i} \mid \kappa_{\alpha^{\prime}} \mu_{q^{\prime}}\right\rangle \\
& \times\left\langle\overrightarrow{\mathrm{k}}_{\rho} m_{j} \mid \kappa_{p} \mu_{p}\right\rangle\left\langle\kappa_{p} \mu_{p} \mid \overrightarrow{\mathrm{k}}, m_{j}\right\rangle\left\langle F\left|\underline{\hat{b}}_{-}^{+} \hat{\hat{b}}_{q^{\prime}}{\underline{\hat{d}_{p}}}_{p}^{+} \hat{d}_{p^{\prime}}\right| F\right\rangle .
\end{aligned}
$$

The expectation value of the spherical wave operators under the sum can be evaluated in the same manner as in Sec. II, and leads to an expression containing the single-particle amplitudes $a_{r q}$ :

$$
\begin{aligned}
\left\langle F\left|\underline{\hat{b}}_{q}^{\dagger} \underline{\hat{b}}_{q^{\prime}}, \underline{\hat{d}}_{p}^{\dagger} \hat{\hat{d}}_{p^{\prime}}\right| F\right\rangle & =\left(\sum_{r<F} a_{r q}^{*} a_{r q^{\prime}}\right)\left(\sum_{s>F} a_{s p} a_{s p^{\prime}}^{*}\right) \\
& -\left(\sum_{r<F} a_{r q}^{*} a_{r p}\right)\left(\sum_{s>F} a_{s q^{\prime}} a_{s p^{\prime}}^{*}\right) .
\end{aligned}
$$

The expression (A7) with (A8) so far is valid quite generally. Now we will restrict our considerations to the monopole approximation and also neglect rotational coupling, i.e., we assume that the amplitudes $a_{r q}$ do not mix between states of different $\kappa$ and $\mu$. This means $\kappa_{r}=\kappa_{q}=\kappa_{q}$, , $\kappa_{s}=\kappa_{p}=\kappa_{p}$, and $\mu_{r}=\mu_{q}=\mu_{q}, \mu_{s}=\mu_{p}=\mu_{p}$, in the first term of Eq. (A8), and $\kappa_{r}=\kappa_{q}=\kappa_{p}, \kappa_{s}=\kappa_{q}$, $=\kappa_{p}$, and $\mu_{r}=\mu_{q}=\mu_{p}, \mu_{s}=\mu_{q}=\mu_{p}$, in the exchange term.

Using the relation

$$
\sum_{\mu}|\langle\mu \kappa \mid \overrightarrow{\mathrm{k}} m\rangle|^{2}=1 / 4 \pi
$$

the direct term in (A7), (A8) can be simplified at once:

$$
\begin{aligned}
N_{i j}^{\prime(\mathrm{di} r)}= & \left.\sum_{q, p}\left|\left\langle\kappa_{q} \mu_{q} \mid \overrightarrow{\mathrm{k}}_{i} m_{i}\right\rangle\right|^{2}\left|\vec{k}_{j} m_{j}\right| \kappa_{b} \mu_{p}\right\rangle\left.\right|^{2} \\
& \times\left(\sum_{r<F}\left|a_{r q}\right|^{2}\right)\left(\sum_{s>F}\left|a_{s p}\right|^{2}\right) \\
= & (4 \pi)^{-2}\left(\sum_{\kappa_{q}} \sum_{r<F}\left|a_{r q}\right|^{2}\right)\left(\sum_{\kappa_{p}} \sum_{s>F}\left|a_{s p}\right|^{2}\right) .
\end{aligned}
$$

This is just the incoherent product of particle and hole probability that also appeared in (A1): The evaluation of the exchange term

$N_{i j}^{(\theta \mathrm{ex})}=-\sum_{q, q^{\prime}}\left\langle\kappa_{q} \mu_{q} \mid \overrightarrow{\mathrm{k}}_{i} m_{i}\right\rangle\left\langle\overrightarrow{\mathrm{k}}_{i} m_{i} \mid \kappa_{q^{\prime}} \mu_{\alpha^{\prime}}\right\rangle\left\langle\overrightarrow{\mathrm{k}}_{j} m_{j} \mid \kappa_{q} \mu_{q}\right\rangle\left\langle\kappa_{q^{\prime}} \mu_{q^{\prime}} \mid \overrightarrow{\mathrm{k}}_{j} m_{j}\right\rangle\left(\sum_{r<F} a_{r, \kappa_{q} E_{i}}^{*} a_{r, \kappa_{q} E_{j}}\right)\left(\sum_{s>F} a_{s, \kappa_{q^{\prime}} E_{i}}^{*} a_{s, k_{q^{\prime}} E_{j}}\right)$

is more tedious. We give the final result, summed over the spin orientations of electron and positron:

$$
\begin{aligned}
N_{\mathbf{k}_{i}, \mathbf{E}_{j}}=\sum_{m_{i}, m_{j}} N_{\overrightarrow{\mathbf{r}}_{i} m_{i}, \overrightarrow{\mathrm{r}}_{j} m_{j}}^{\prime}=(4 \pi)^{-2} & \left\{4\left(N_{E_{i}}^{(-)}+N_{E_{i}}^{(+)}\right)\left(\bar{N}_{E_{j}}^{(-)}+\bar{N}_{E_{j}}^{(+)}\right)+2\left|\sum_{r<F} a_{r, E_{i}}^{(-)} a_{r, E_{j}}^{(-)}\right|^{2}+2\left|\sum_{r<F} a_{r, E_{i}}^{(+)} a_{r, E_{j}}^{(+)}\right|^{2}\right. \\
& \left.+4 \cos \theta_{i j} \operatorname{Re}\left[e^{i \Delta}\left(\sum_{r<F} a_{r, E_{i}}^{(-) *} a_{r, E_{j}}^{(-)}\right)\left(\sum_{r<F} a_{r, E_{i}}^{(+) *} a_{r, E_{j}}^{(+)}\right)\right]\right\} .
\end{aligned}
$$

Here $\Delta$ is the relative phase $\Delta=\left(\delta_{i}^{(-)}-\delta_{j}^{(-)}\right)-\left(\delta_{i}^{(+)}-\delta_{j}^{(+)}\right)$and $\theta_{i j}$ is the angle between $\overrightarrow{\mathrm{k}}_{i}$ and $\overrightarrow{\mathrm{k}}_{j}$. In perturbation theory (A12) reduces to

$$
N_{E_{i}, \dot{\mathrm{r}}_{j}}^{\prime} \simeq 2(4 \pi)^{-2}\left(\left|a_{E_{j}, E_{i}}^{(-)}\right|^{2}+\left|a_{E_{j}, E_{i}}^{(+)}\right|^{2}+2 \cos \theta_{i j} \cos \Delta\left|a_{E_{j}, E_{i}}^{(-)}\right|\left|a_{E_{j,} E_{i}}^{(+)}\right|\right)
$$

Upon integration over the angles, the last two equation reduce to (A1) and (A2).

Equation (A12) shows, that the emitted pair has an angular correlation that results from the interference 
between $\kappa=+1$ and -1 waves. We do not expect, however, that this effect will lead to significant structures as a function of positron energy in supercritical collisions. The phase shift $\Delta$ has to be determined for the final wave function. In contrast to the (academic) problem of monopole pair conversion in a stationary supercritical atom, $\Delta$ does not exhibit a resonance behavior in the case of a heavy-ion collision. Moreover, the relative magnitude of the interference term is smaller than in the case of pair conversion for the reasons discussed earlier.

*Permanent address: Institut für Theoretische Physik, Johann Wolfgang Goethe Universität, 6000 Frankfurt am Main, West Germany.

${ }^{1}$ The subject of QED of strong fields has been reviewed in Refs. 2-5 where an extensive bibliography can also be found.

${ }^{2}$ B. Müller, Annu. Rev. Nucl. Sci. 26, 351 (1976).

${ }^{3} \mathrm{~J}$. Reinhardt, W. Greiner, Rep. Prog. Phys. 40, 219 (1977).

${ }^{4}$ J. Rafelski, L. P. Fulcher, and A. Klein, Phys. Rep. 38C, 227 (1978).

${ }^{5}$ S. J. Brodsky and P. J. Mohr, in Structure and Collisions of Ions and Atoms, edited by I. A. Sellin (Springer, Berlin, Heidelberg, and New York, 1978), p. 3.

${ }^{6}$ B. Müller, J. Rafelski, and W. Greiner, Z. Phys. 257, 62 (1972); 257, 183 (1972).

${ }^{7}$ Y. B. Zeldovich and V. S. Popov, Usp. Fiz. Nauk 105, 403 (1971) [Sov. Phys._Usp. 14, 673 (1972)].

${ }^{8}$ O. Klein, Z. Phys. 53, 157 (1929).

${ }^{9}$ F. Hund, Z. Phys. $\overline{117}, 1$ (1941).

${ }^{10} \mathrm{~W}$. Pieper and W. Greiner, Z. Phys. 218, 126 (1969).

${ }^{11}$ S. S. Gershtein and Y. B. Zeldovich, Zh. Eksp. Teor. Fiz. 57, 654 (1969) [Sov. Phys.-JETP 30, 358 (1970)].

${ }^{12}$ B. Müller, H. Peitz, J. Rafelski, and W. Greiner, Phys. Rev. Lett. 28, 1235 (1972).

${ }^{13} \mathrm{~J}$. Schwinger, Phys. Rev. 82,664 (1951).

${ }^{14}$ V. S. Popov, Yad. Fiz. 12, 429 (1970) [Sov. J. Nucl. Phys. 12, 235 (1971)].

${ }^{15} \mathrm{G}$. Soff, B. Müller, and J. Rafelski, Z. Naturforsch. 29a, 1267 (1974).

${ }^{16}$ Earlier studies of this problem are (a) I. Pomeranchuk and J. Smorodinsky, J. Phys. (Moscow) 9, 97 (1945); (b) F. G. Werner and J. A. Wheeler, Phys. Rev. 109, 126 (1958); (c) V. V. Voronkov and N. N. Koleznikov, Zh. Eksp. Teor. Fiz. 39, 189 (1960) [Sov. Phys.-JETP $12,136(1961)]$.

${ }^{17} \mathrm{~J}$. Rafelski, B. Müller, and W. Greiner, Nucl. Phys. B 68, 585 (1974).

${ }^{18}$ L. P. Fulcher and A. Klein, Phys. Rev. D $\underline{8}, 2455$ (1973).

${ }^{19}$ P. Gärtner, B. Müller, J. Reinhardt, and W. Greiner, Phys. Lett. 95B, 181 (1980).

${ }^{20} \mathrm{~W}$. Heitler and L. Nordheim, J. Phys. (Paris) $\underline{5}, 449$ (1934).

${ }^{21}$ E. Lifshitz, Phys. Z. Sowjetunion 7, 385 (1935).

${ }^{22}$ W. E. Stephens and H. Staub, Phys. Rev. 109, 1196 (1958).

${ }^{23}$ M. Heykants and M. Niecke, Nucl. Phys. A $\underline{332}, 22$ (1979).

${ }^{24} \mathrm{~J}$. Bang and J. M. Hansteen, K. Dan. Vidensk. Selsk., Mat. Fys. Medd. 31 , No. 13 (1959); J. M. Hansteen, Adv. At. Mol. Phys. 11, 299 (1975).
${ }^{25}$ E. Hara, Z. Naturforsch. 16a, 155 (1965).

${ }^{26} \mathrm{~J}$. Reinhardt, B. Müller, and W. Greiner, in Coherence and Correlation in Atomic Collisions, edited by H. Kleinpoppen and J. F. Williams (Plenum, New York, 1979), p. 331.

${ }^{27} J$. Reinhardt, G. Soff, B. Müller, and W. Greiner, Prog. Part. Nucl. Phys. 4, 503 (1980).

${ }^{28}$ N. S. Mott and H. S. W. Massey, The Theory of Atomic Collisions, 3rd ed. (Oxford University Press, Oxford, 1965).

${ }^{29} \mathrm{D}$. H. Madison and E. Merzbacher, in Atomic InnerShell Processes, edited by B. Crasemann (Academic, New York, 1975), Vol. I, p. 1.

${ }^{30}$ J. S. Briggs, Rep. Prog. Phys. 39, 217 (1976).

${ }^{31} \mathrm{~J}$. Rafelski and B. Müller, Phys. Rev. Lett. 36, 517 (1976).

${ }^{32}$ D. Kolb, W.-D. Sepp, B. Fricke, and T. Morovic, Z. Phys. A286, 169 (1978).

${ }^{33}$ G. Soff, J. Reinhardt, and W. Greiner, Phys. Rev. A 23, 701 (1981).

${ }^{34}$ V. Maruhn-Rezwani, N. Grün, and W. Scheid, Phys. Rev. Lett. $\underline{43}, 512$ (1979).

${ }^{35}$ J. S. Briggs, J. Phys. B 8, L485 (1975).

${ }^{36} \mathrm{~J}$. Rafelski, B. Müller, and W. Greiner, Lett. Nuovo Cimento 4, 469 (1972).

${ }^{37}$ J. Theis, J. Reinhardt, and B. Müller, J. Phys. B 12, L479 (1979).

${ }^{38}$ T. A. Green, Proc. Phys. Soc. London 86, 1017 (1965); R. Shakeshaft, J. Macek, and E. Gerjuoy, J. Phys. B 6, 794 (1973).

${ }^{39}$ B. Müller and V. E. Oberacker, Phys. Rev. C $\underline{22}, 1909$ (1980).

${ }^{40} \mathrm{~J}$. F. Reading, Phys. Rev. A 8,3262 (1973).

${ }^{41} \mathrm{~J}$. Reinhardt, B. Müller, W. Greiner, and G. Soff, Phys. Rev. Lett. $\underline{43}, 1307$ (1979).

${ }^{42} \mathrm{~J}$. F. Reading and A. L. Ford, Phys. Rev. A 21, 124 (1980).

${ }^{43}$ G. Soff, J. Reinhardt, B. Müller, and W. Greiner, Z. Phys. A294, 137 (1980).

${ }^{44} \mathrm{H}$. Peitz, B. Müller, J. Rafelski, and W. Greiner, Lett. Nuovo Cimento $\underline{8}, 37$ (1973).

${ }^{45}$ S. S. Gershtein and V. S. Popov, Lett. Nuovo Cimento 6, 593 (1973); V. S. Popov, Zh. Eksp. Teor. Fiz. 65 , $\overline{3} 5$ (1973) [Sov. Phys.—JETP 38, 18 (1974)]; Yad. Fiz. 19,155 (1974) [Sov. J. Nucl. Phys. 19, 81 (1974)]; M. S. Marinov and V. S. Popov, Yad. Fiz. 20,1223 (1974) [Sov. J. Nucl. Phys. 20, 641 (1975)].

${ }^{46} \mathrm{~J}$. Reinhardt, Ph.D. thesis, Frankfurt, 1979 (unpublished).

${ }^{47}$ W. L. Wang and C. M. Shakin, Phys. Lett. $\underline{32 B}, 421$ (1970).

${ }^{48} \mathrm{H}$. W. Barz, I. Rotter, and J. Höhn, Phys. Lett. 37B, 4 (1971).

${ }^{49}$ W. L. Wang and C. M. Shakin, Phys. Rev. C $\underline{5}, 1898$ 
(1972).

${ }^{50}$ N. Auerbach, J. Hüfner, A. K. Kerman, and C. M. Shakin, Rev. Mod, Phys. 44, 48 (1972).

${ }^{51}$ I. Rotter, H.-W. Barz, R. Wünsch, and J. Höhn, Part. Nucl. (USSR) 6, 435 (1975).

${ }^{52}$ M. Micklinghoff, Ph.D. thesis, Hamburg, 1977 (unpublished); Nucl. Phys. A295, 228 (1978).

${ }^{53}$ H. Feshbach, Ann. Phys. (N.Y.) 19, 287 (1962).

${ }^{54}$ See, e.g., M. Gyulassy, Nucl. Phys. A244, 497 (1975).

${ }^{55}$ A. Sandulescu, I. Silisteanu, and R. Wünsch, Nucl. Phys. A305, 205 (1978).

${ }^{56}$ G. Soff, W. Betz, J. Reinhardt, and J. Rafelski, Phys. Scr. 17, 417 (1978).

${ }^{57}$ B. Müller, J. Rafelski, and W. Greiner, Nuovo Cimento A18, 551 (1973).

${ }^{58}$ B. Müller, J. Rafelski, and W. Greiner, Phys. Lett. 47B, 5 (1973); B. Müller and W. Greiner, Z. Naturforsch. 31a, 1 (1976).

${ }^{59} \mathrm{~J}$. Rafelski and B. Muller, Phys. Lett. 65B, 205 (1976).

${ }^{60} \mathrm{~W}$. Betz, Ph.D. thesis, Frankfurt, 1980 (unpublished).

${ }^{61} \mathrm{G}$. Soff, W. Greiner, W. Betz, and B. Müller, Phys. Rev. A 20, 169 (1979).

${ }^{62}$ D. R. Bates and R. Mc Carroll, Proc. R. Soc. London A245, 311 (1958).

${ }^{63}$ S. B. Schneidermann and A. Russek, Phys. Rev. 181 311 (1969).

${ }^{64} \mathrm{H}$. Levy II and W. R. Thorson, Phys. Rev. 181, 244 (1969); 181, 252 (1969).

${ }^{65}$ G. B. Schmid, Phys. Rev. A 15, 1459 (1977).

${ }^{66}$ W. R. Thorson and J. B. Delos, Phys. Rev. A $\underline{18}, 117$ (1978).

${ }^{67} \mathrm{~J}$. Vaaben and $\mathrm{K}$. Taulbjerg (unpublished).

${ }^{68} \mathrm{U}$. Heinz, W. Greiner, and B. Müller, Phys. Rev. A 23,562 (1981).

${ }^{69}$ T. H. Rihan, N. S. Aly, E. Merzbacher, B. Müller, and W. Greiner, Z. Phys. A285, 397 (1978).

${ }^{70}$ W.-D. Sepp, B. Fricke, and T. Morovic, Phys. Lett. $81 \mathrm{~A}, 258$ (1981).

${ }^{71}$ T. de Reus, diploma thesis, Frankfurt, 1981 (unpublished).

${ }^{72}$ V. I. Lisin, M. S. Marinov, and V. S. Popov, Phys. Lett. 69B, 141 (1977).

${ }^{73}$ V. I. Lisin and M. S. Marinov, Phys. Lett. $\underline{91 B}, 20$ (1980).

${ }^{74}$ K.-H. Wietschorke, B. Müller, W. Greiner, G. Soff, J. Phys. B 12, L31 (1979).

${ }^{75} \mathrm{G}$. Soff, J. Reinhardt, B. Müller, and W. Greiner, Phys. Rev. Lett. 38, 592 (1977).

${ }^{76}$ B. Müller, G. Soff, W. Greiner, and V. Ceausescu, $Z$. Phys. A285, 27 (1978).

${ }^{77} \mathrm{E}$. Kankeleit, Nukleonika 25, 253 (1980).

${ }^{78}$ F. Bosch, D. Liesen, P. Armbruster, D. Maor, P. H. Mokler, H. Schmidt-Böcking, and R. Schuch, Z. Phys. A296, 11 (1980).

${ }^{79} \mathrm{~J}$. Bang and J. M. Hansteen, Phys. Lett. 72A, 218 (1979).

${ }^{80} \mathrm{~J}$. S. Greenberg, H. Bokemeyer, E. Emling, E. Grosse, D. Schwalm, and F. Bosch, Phys. Rev. Lett. 39, 1404 (1977).

${ }^{81}$ D. Liesen, P. Armbruster, H.-H. Behncke, and S. Hagmann, Z. Phys. A288, 417 (1978).

${ }^{82}$ J. R. MacDonald, P. Armbruster, H.-H. Behncke, F. Folkmann, S. Hagmann, D. Liesen, P. H. Mokler, and A. Warczak, Z. Phys. A284, 57 (1978).

${ }^{83}$ H.-H. Behncke, D. Liesen, S. Hagmann, P. H. Mokler, and P. Armbruster, Z. Phys. A288, 35 (1978).

${ }^{84}$ R. Anholt and W. E. Meyerhof, Phys. Lett. 64A, 381 (1978).

${ }^{85}$ R. Anholt, W. E. Meyerhof, and Ch. Stoller, Z. Phys. A291, 287 (1979).

${ }^{86}$ D. Liesen, P. Armbruster, F. Bosch, S. Hagmann, P. H. Mokler, H. Schmidt-Böcking, R. Schuch, J. B. Wilhelmy, and H. J. Wollersheim, Phys. Rev. Lett. 44, 983 (1980).

${ }^{87} \mathrm{H}$. Backe, L. Handschug, F. Hessberger, E. Kankeleit, L. Richter, F. Weik, R. Willwater, H. Bokemeyer, P. Vincent, Y. Nakayama, and J. S. Greenberg, Phys. Rev. Lett. $\underline{40}, 1443$ (1978).

${ }^{88} \mathrm{C}$. Kozhuharov, P. Kienle, E. Berdermann, H. Bokemeyer, J. S. Greenberg, Y. Nakayama, P. Vincent, H. Backe, L. Handschug, and E. Kankeleit, Phys. Rev. Lett. 42, 376 (1979).

${ }^{89}$ H. Backe, W. Bonin, W. Engelhardt, E. Kankeleit, V. Metag, and J. B. Wilhelmy (unpublished); H. Backe, W. Bonin, W. Engelhardt, E. Kankeleit, M. Mutterer, P. Senger, F. Weik, R. Willwater, V. Metag, and J. B. Wilhelmy (unpublished).

${ }^{90} \mathrm{~A}$. Balanda, K. Bethge, H. Bokemeyer, H. Folger, J. S. Greenberg, H. Grein, A. Gruppe, S. Ito, S. Matsuki, R. Schule, D. Schwalm, J. Schweppe, P. Vincent, and M. Waldschmidt (unpublished).

${ }^{91}$ P. Armbruster and P. Kienle, Z. Phys. A291, 399 (1979).

${ }^{92} \mathrm{~J}$. Reinhardt, V. Oberacker, B. Müller, W. Greiner, and G. Soff, Phys. Lett. 78B, 183 (1978).

${ }^{93}$ Second-order perturbation theory recently has also been considered in a (nonrelativistic) analytical calculation using a united atom picture by J. Bang and J. M. Hansteen, Phys. Scr. 22, 609 (1981).

${ }^{94}$ W. Betz, G. Soff, B. Müller, and W. Greiner, Phys. Rev. Lett. 37, 1046 (1976).

${ }^{95} \mathrm{G}$. Soff, W. Betz, B. Müller, W. Greiner, and E. Merzbacher, Phys. Lett. 65A, 19 (1978); G. Soff, B. Müller, and W. Greiner, Phys. Rev. Lett. 40 , 540 (1978).

${ }^{96} \mathrm{~J}$. Rafelski, B. Müller, and W. Greiner, Z. Phys. A285, 49 (1978).

${ }^{97}$ U. Müller, J. Reinhardt, B. Müller, and W. Greiner (unpublished).

${ }^{98}$ The authors of Ref. 99 maintain the view, that changes in the $S$ matrix and not the gauge- and frame-dependent energy eigenvalues are the relevant observables. According to our results a relatively small spreading of tive time scale will produce such changes.

${ }^{99}$ H. Fierz and G. Scharf, Helv. Phys. Acta 52, 437 (1980).

${ }^{100}$ The use of the nuclear blocking effect has been suggested by K. G. Kaun, S. A. Karamyan, Report No. JINR-P7-11420, 1978 (unpublished).

${ }^{101}$ P. Schlüter, G. Soff, and W. Greiner, Z. Phys. A286, 149 (1978); P. Schlüter, G. Soff, and W. Greiner, Phys. Rep. (in press).

${ }^{102}$ V. Oberacker, G. Soff, and W. Greiner, Phys. Rev. Lett. 36, 1024 (1976); Nucl. Phys. A259, 324 (1976).

${ }^{103}$ W. E. Meyerhof, R. Anholt, Y. El Masry, I. Y. Lee, D. Cline, F. S. Stephens, and R. M. Diamond, Phys. Lett. $\underline{69 B}, 41$ (1977). 
${ }^{104} \mathrm{H}$. Backe (private communication)

${ }^{105} \mathrm{P}$. Vincent (private communication).

${ }^{106} \mathrm{C}$. Kozhuharov (private communication).

${ }^{107} \mathrm{G}$. A. Rinker and L. Wilets, Phys. Rev. Lett. 31, 1559

(1973); M. Gyulassy, Phys. Rev. Lett. 33, 921 (1974).

${ }^{108}$ K.-T. Cheng and W. R. Johnson, Phys. Rev. A $\underline{14}$,

1943 (1976).

${ }^{109} \mathrm{E}$. Kankeleit (private communication).

${ }^{110}$ L. C. Biedenharn and M. E. Rose, Rev. Mod. Phys.

25, 729 (1953).

${ }^{111}$ M. E. Rose, Relativistic Electron Theory (Wiley, New York, 1961). 